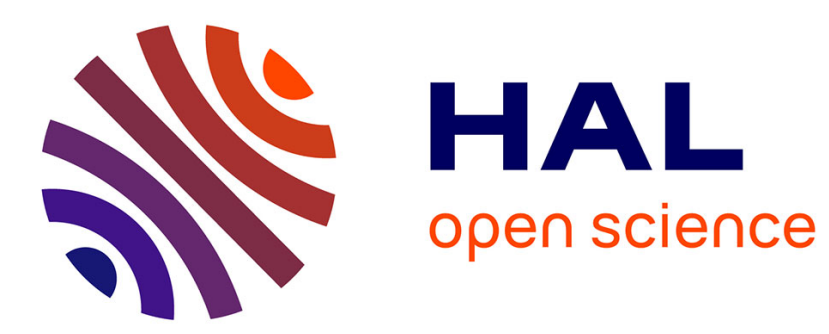

\title{
Finite element implementation of the coupled criterion for numerical simulations of crack initiation and propagation in brittle materials
}

\author{
Jia Li, Dominique Leguillon
}

\section{- To cite this version:}

Jia Li, Dominique Leguillon. Finite element implementation of the coupled criterion for numerical simulations of crack initiation and propagation in brittle materials. Theoretical and Applied Fracture Mechanics, 2017, 93, pp.105-115. 10.1016/j.tafmec.2017.07.010 . hal-01578011

\section{HAL Id: hal-01578011 https://hal.sorbonne-universite.fr/hal-01578011}

Submitted on 28 Aug 2017

HAL is a multi-disciplinary open access archive for the deposit and dissemination of scientific research documents, whether they are published or not. The documents may come from teaching and research institutions in France or abroad, or from public or private research centers.
L'archive ouverte pluridisciplinaire HAL, est destinée au dépôt et à la diffusion de documents scientifiques de niveau recherche, publiés ou non, émanant des établissements d'enseignement et de recherche français ou étrangers, des laboratoires publics ou privés. 


\section{Accepted Manuscript}

Finite element implementation of the coupled criterion for numerical simulations of crack initiation and propagation in brittle materials

Jia Li, Dominique Leguillon

PII:

S0167-8442(17)30029-0

DOI: http://dx.doi.org/10.1016/j.tafmec.2017.07.010

Reference:

TAFMEC 1915

To appear in:

Theoretical and Applied Fracture Mechanics

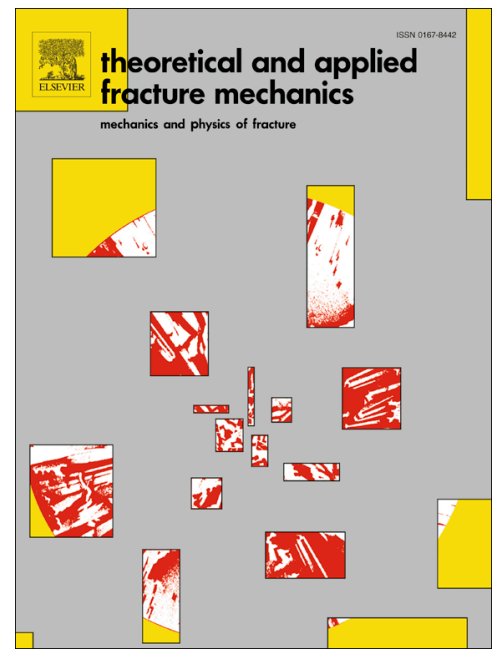

Received Date: $\quad 18$ January 2017

Revised Date: 6 July 2017

Accepted Date: 7 July 2017

Please cite this article as: J. Li, D. Leguillon, Finite element implementation of the coupled criterion for numerical simulations of crack initiation and propagation in brittle materials, Theoretical and Applied Fracture Mechanics (2017), doi: http://dx.doi.org/10.1016/j.tafmec.2017.07.010

This is a PDF file of an unedited manuscript that has been accepted for publication. As a service to our customers we are providing this early version of the manuscript. The manuscript will undergo copyediting, typesetting, and review of the resulting proof before it is published in its final form. Please note that during the production process errors may be discovered which could affect the content, and all legal disclaimers that apply to the journal pertain. 
Highlighted version

\title{
Finite element implementation of the coupled criterion for numerical simulations of crack initiation and propagation in brittle materials
}

\author{
$\mathrm{Jia} \mathrm{Li}^{\mathrm{a}^{*}}$, Dominique Leguillon ${ }^{\mathrm{b}}$
}

${ }^{a}$ LSPM, CNRS UPR 3407, Institut Galilée, Université Sorbonne Paris Cité, Université Paris 13, F93430 Villetaneuse, France

${ }^{\mathrm{b}}$ Institut Jean Le Rond d'Alembert, CNRS UMR 7190, Sorbonne Universités, UPMC Université Paris 06, F-75005 Paris, France.

*: Corresponding author

LSPM, CNRS UPR 3407

Institut Galilée, Université Paris 13

99 Avenue Jean-Baptiste Clément, 93430 Villetaneuse, France

Tel : $33149402889 \quad$ Fax : 33149403938

E-mail : jia.li@univ-paris13.fr

\begin{abstract}
The proposed numerical model for simulating two-dimensional cracking process in brittle materials is based on the coupled criterion (CC) which enters in the general framework of Finite Fracture Mechanics. It states that both the energy and stress conditions should be fulfilled to predict the crack creation in brittle solids. Special numerical approaches were developed in drawing correctly the potential crack paths and to minimize the potential energy with respect to all predicted crack paths. The originality of this work lies in the ability to describe both the initiation and the growth of one or more cracks. Moreover, statistical distributions of flaws can be accounted for. The efficiency and accuracy of the proposed approach were approved by means of several numerical examples for which the solutions are well-known or well-studied in the literature.
\end{abstract}

Keywords: Crack initiation, crack growth, multiple cracking, finite element analysis, brittle or quasi-brittle materials.

\section{Introduction}

Numerical prediction of fracture in solids is a classical challenge for mechanics and computational sciences. Developing efficient and accurate numerical models to simulate fracture behavior has been extensively studied in the last two decades. Even though numerous theoretical and numerical studies exist in the literature in predicting the crack growth, few numerical models are available to predict both the crack initiation and crack growth. 
Among these models, the most popular and efficient one nowadays is the cohesive zone model (CZM) [1-2]. A wide range of phenomena have been analyzed using this framework. Numerical schemes, like the finite element method, have become the mainstay for solution involving CZM. Crack propagation - crack nucleation can be modeled by the introduction of cohesive surfaces [3-4] allowing for shear as well as tensile debonding. One advantage of this type of formulation is that it no longer requires an initial crack, whose length and direction is difficult to prescribe, so that crack nucleation can easily be analyzed. Generally speaking, the cohesive elements can be inserted between all bulk elements such that the cohesive model can select automatically the crack paths. However, if the finite element mesh is refined and cohesive surfaces are continually added between all elements, convergence cannot always be expected. An additional result is logically the expensive computational costs. Practically, the cohesive elements are only inserted at predefined crack paths to avoid these numerical complications. These points have been well documented in the literature [5-6].

Apart from the CZM, the so-called phase-field model provides another possibility to predict both the crack initiation and crack growth. Francfort and Marigo [7] developed a so-called damage gradient model in which the damage zone converges to a crack as a prescribed length parameter tends to zero. Efficient algorithms were developed in order to solve complicated cracking problems [8-9]. Recently, phase-field models have found a widespread popularity for simulating brittle crack initiation and propagation in a smeared manner. However, concerns have been raised as well. It has been shown that the load-displacement curves can depend considerably on the internal length scale $l$ that defines the width of the distributed fracture zone and on the degradation function that has been introduced [10-11]. Once the parameters $G_{c}$, the critical energy release rate, and $l$ are specified according to the material under consideration and the mesh density is given, there is no room left to specify the critical stress [12].

In studying the crack onset from the root of a sharp V-notch, Leguillon [13] noticed that application of a single conventional fracture criterion, namely the Griffith energy criterion or the stress criterion, is not suitable and often leads to paradoxical predictions. It was concluded that when fracture occurs both criteria are fulfilled simultaneously, even if one often hides the other. Both are necessary conditions and together they form a sufficient one. Application of this coupled criterion (CC) to crack onset predictions at a V-notch root shows remarkably satisfactory agreement with respect to experimental results. Afterward, the CC gets increasing popularity in fracture prediction of brittle or quasi-brittle materials. A large number of fracture cases were studied by using this criterion [14-19 among others].

If the accuracy and efficiency of this criterion were approved by means of numerous real structure analyses, these studies were often performed case by case by using particular 
resolution techniques such as asymptotic analysis, local finite element modelling etc. Therefore, we believe that it is necessary and useful to implement this criterion into a numerical code such that more complicated fracture problems can be dealt with. This is the main motivation of the present work.

Herein, various numerical efforts were made for developing algorithms to predict automatically the location of crack nucleation and next their path. By means of these efforts, the $\mathrm{CC}$ was successfully implemented into a finite element code with which two-dimensional fractures in brittle materials can readily be simulated. Moreover, several classical fracture problems were studied in order to assess the efficiency of the proposed numerical model. The numerical results were compared with some well-known solutions in the literature to show that they are fully satisfactory.

\section{Description of the numerical fracture model}

\subsection{The coupled criterion}

The coupled criterion (CC) was widely presented in the literature (see the review paper by Weissgraeber et al. [20]). Herein we just give a brief outline. The CC is based on the Finite Fracture Mechanics (FFM) in which the Griffith criterion is written in incremental form:

$$
-\frac{\delta \Pi}{\delta S}=G^{i n c} \geq G_{c}
$$

where $\delta \Pi$ is the change in potential energy, $\delta S=t \delta l$ is the newly created crack surface (within the $2 \mathrm{D}$ framework $t$ is the thickness of the body under consideration and $\delta l$ the crack increment length), $G^{i n c}$ is the so-called incremental energy release rate and $G_{c}$ is its critical value representing the fracture toughness of the material. This criterion is not sufficient since it depends on the newly created surface $\delta S$ (up to now unknown). In order to remedy this shortcoming, the stress condition has to be added. For brittle materials, the maximal principal stress criterion is an appropriate candidate:

$$
\sigma_{1} \geq \sigma_{c}
$$

where $\sigma_{1}$ and $\sigma_{c}$ are respectively the maximal principal stress and the ultimate stress of the material. Within a plane elasticity framework, Leguillon [13] states that new crack is created if and only if both the stress and energy conditions are verified, i.e., the stress criterion is satisfied at all the points along the newly created crack path, i.e. over whole $\delta S$, and the Griffith criterion is satisfied with the corresponding crack path.

One of the main advantages of the $\mathrm{CC}$ is its ability to predict both the crack initiation and crack growth. In fact, through a matched asymptotic expansion analysis Leguillon [13] showed that the applied stress $\sigma_{a p p}^{*}$ at crack initiation can be written as 


$$
\sigma_{a p p}^{*}=E\left(\frac{G^{c}}{G_{0}}\right)^{1-\lambda}\left(\frac{\sigma^{c}}{\sigma_{0}}\right)^{2 \lambda-1}
$$

where $\lambda(<1)$ is the singularity exponent of the stress concentration (in case of a V-notch for instance), $\left(\sigma_{0}, G_{0}\right)$ are scaling coefficients. It is clear that this formulation coincides with the Griffith criterion in the presence of a crack $(\lambda=0.5)$ and with the strength criterion for a straight edge $(\lambda=1)$.

The CC is conceptually clear and physically convincing. Its application to different academic or engineering fracture problems has shown remarkable efficiency and accuracy. It is easy to apply when the fracture locations and the crack paths are previously known. However, these data are not always available for complex structures. Consequently, the first task in a numerical implementation of this criterion is to determine the locus of the crack creation. To this end, some simple but physically reasonable hypotheses have been adopted. In the following, we present the numerical details in the implementation of this criterion into a FE code. The three-node linear triangle elements are used in the simulations. Several reasons prompt us to use such a simple element. First, the adopted procedure of representing crack growth by element elimination requires very strong mesh refinements, thus it is convenient to use the simplest finite-element diminishing in this way high computational costs. Second, the usage of constant-stress finite-element allows a simple implementation of the procedure for crack path prediction proposed in Section 2.4. Finally, an increase of the degree of interpolation is useless in the vicinity of an elastic singularity because of the lack of smoothness of the solution.

\subsection{General computational consideration}

In the proposed numerical model, the external loads are applied in an incremental manner. According to the $\mathrm{CC}$, one of the necessary conditions of fracture is that the stress criterion $\sigma_{1} / \sigma_{c} \geq 1$ must be fulfilled all along the supposed crack paths. Consequently, we should firstly find all the zones in which $\sigma_{1} / \sigma_{c} \geq 1$, each containing one and only one local maximum of $\sigma_{1}$. We further assume that only one crack is created in each of such zones. If no such a zone is detected, the applied loads are incrementally increased.

The cracks may potentially initiate from the point where the first principal stresses $\sigma_{1}$ is locally maximum and propagates along the direction perpendicular to the first principal direction. Among all these potential cracks, the real cracks will be the combination making the incremental energy release rate maximum. This hypothesis is a classical one in fracture mechanics [21] even though the stress criterion is not considered. Furthermore, in the framework of FFM, Mantic [22] formulated mathematically this hypothesis as a minimization problem. All these hypotheses allow building a robust computational algorithm for the assessment of both cracks initiation and further cracks growth. 


\subsection{Locations of the potential newly created cracks.}

According to the above-mentioned general consideration, the first task is to find the zones in which $\sigma_{1} / \sigma_{c} \geq 1$, each containing a local maximum of $\sigma_{1}$ under a given prescribed load.

To this end, we inverse the sign of $\sigma_{1}$, such that a local maximum value become a local minimum value. Then we use the so-called watershed flooding technique to find these zones. The basic idea consists in considering the stress distribution as a topographic surface and placing a water source in each local minimum of its relief. Next the entire relief is gradually flooded from the sources and dams are placed where the different water sources meet. Figure 1 shows the flooding process of a 1D surface according to which the algorithm was built [23].

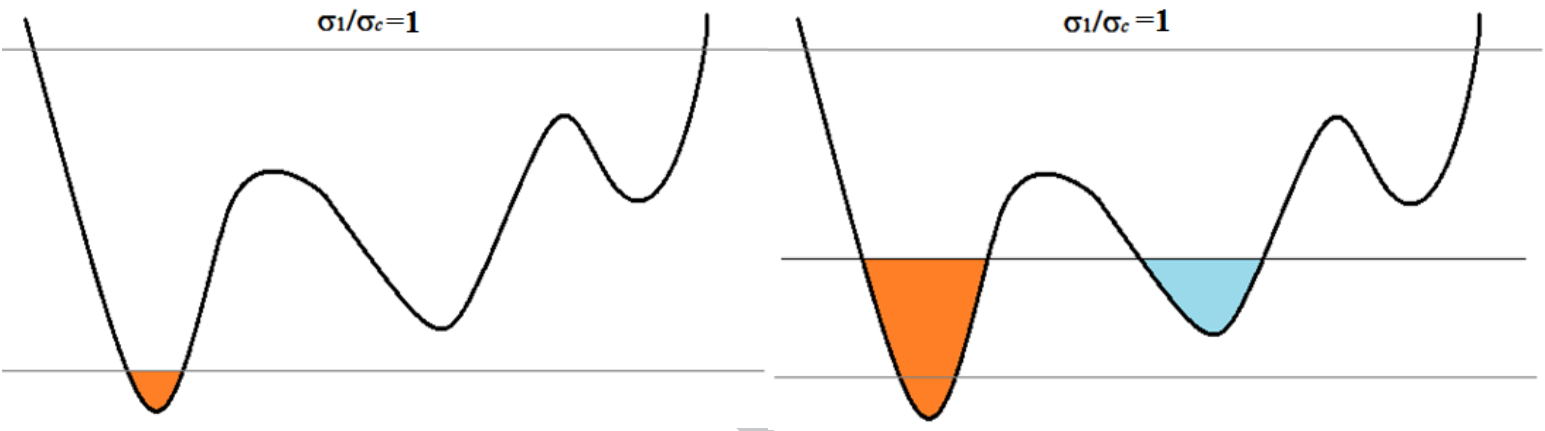

(a)

(b)

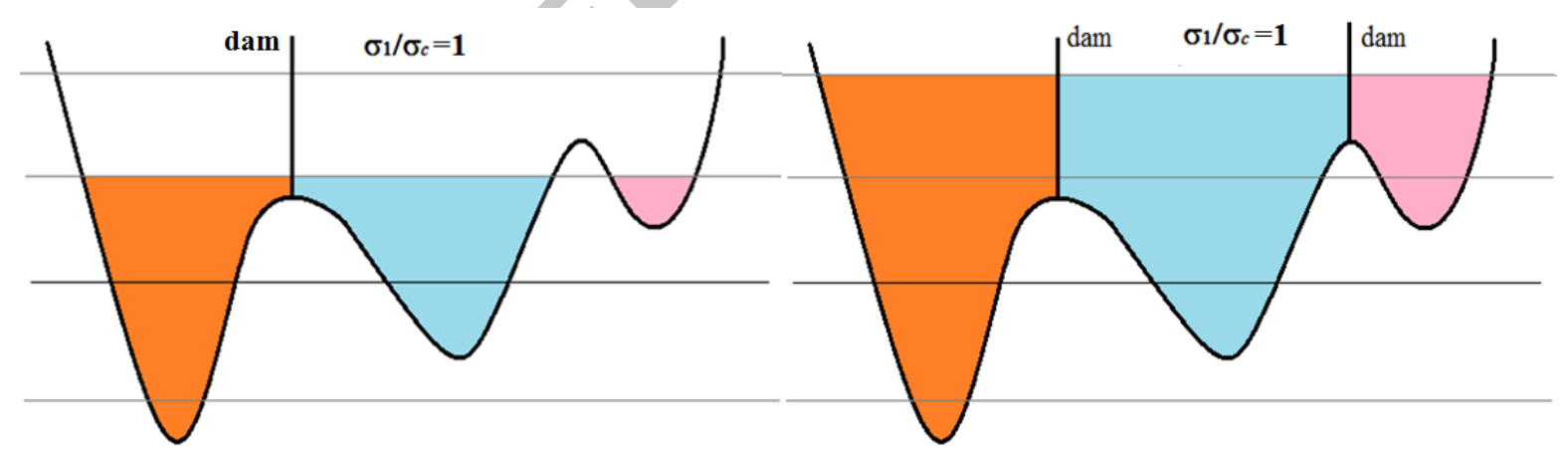

(c)

(d)

Figure 1: Watershed flooding process (a): a water source is placed at the point where $-\sigma_{1} / \sigma_{c}$ is minimum, increasing the water level allows finding the neighboring elements and then creating the first catchment basin; (b) by increasing the water level, another distinguished local minimum is found from which the second catchment basin is created; (c) when the first and second catchment basins meet, a dam is placed; (d) continuing this process until $\sigma_{1} / \sigma_{c}=1$ allows finding distinct catchment basins containing each a local minimum of $-\sigma_{1} / \sigma_{c}$.

All elements in the zone around a given minimum constitute the catchment basin associated with that minimum. The watersheds are the zones dividing adjacent catchment basins. The 
first points that are reached by water are the elements at the highest stress value, i.e., points where $-\sigma_{1} / \sigma_{c}$ are minimal, and then all elements are progressively reached up to the stress level $\sigma_{1} / \sigma_{c}=1$.

The catchment basins are labeled with integer values. Each catchment basin contains a local maximum $\sigma_{1}$ from which a potential crack can be created.

Normally, the classical watershed technique often lead to over-segmentation phenomenon, which consists in finding out too many catchment basins due to noisy numerical results. There exist numerous denoising techniques in the literature to reduce this over-segmentation [24]. In this work, we just pre-process the topographic surface by using the median filtering technique for its simplicity. The main idea of the median filter is to run through the stress distribution map element by element, replacing the $\sigma_{1}$ value of the element with the median value of neighboring elements. In the case when the stress evaluation is sufficiently accurate, this simple technique is sufficient to reduce efficiently the over-segmentation phenomenon.

This algorithm allows extracting $N$ zones $\mathbf{S}_{n}$, each of them containing a local maximum of the first principal stress like a "hill peak".

\subsection{Drawing the potential crack paths}

In each zone $\mathbf{S}_{n}$ a potential crack can be created. According to the maximal principal stress criterion, the direction of the newly created crack is perpendicular to the first principal stress. This criterion permits to draw a principal stress trajectory from each "hill peak". The following algorithm is used to determine the crack paths:

1. From the point $\left(x_{0}, y_{0}\right)$ corresponding to the "hill peak" in $\mathbf{S}_{n}$, we draw first the right branch of the predicted crack path. To this end, we calculate the coordinates $x_{1}=x_{0}+\Delta l \cos \alpha_{0}, y_{1}=y_{0}+\Delta l \sin \alpha_{0}$, where $\alpha_{0}$ is the angle of the first principal stress trajectory at the point $\left(x_{0}, y_{0}\right)$. Varying $\Delta l$ if necessary such that the point $\left(x_{1}, y_{1}\right)$ is located in an adjacent element;

2. If the point $\left(x_{1}, y_{1}\right)$ is not located in $\mathbf{S}_{n}$, go to step 5. Else from the point $\left(x_{1}, y_{1}\right)$ and with the angle of the first principal stress trajectory $\alpha_{1}$ at this point, calculate

$x_{2}^{\text {right }}=x_{1}+\Delta l \cos \alpha_{1}, y_{2}^{\text {right }}=y_{1}+\Delta l \sin \alpha_{1}$ and

$x_{2}^{\text {left }}=x_{1}-\Delta l \cos \alpha_{1}, y_{2}^{\text {left }}=y_{1}-\Delta l \sin \alpha_{1}$

Varying $\pm \Delta l$ if necessary such that the points $\left(x_{2}^{\text {right }}, y_{2}^{\text {right }}\right)$ and $\left(x_{2}^{\text {left }}, y_{2}^{\text {left }}\right)$ are located in the adjacent elements;

3. Let $x_{2}=x_{2}^{\text {right }}, y_{2}=y_{2}^{\text {right }}$ if $\sigma_{1}\left(x_{2}^{\text {right }}, y_{2}^{\text {right }}\right)>\sigma_{1}\left(x_{2}^{\text {left }}, y_{2}^{\text {left }}\right)$, else let $x_{2}=x_{2}^{\text {left }}, y_{2}=y_{2}^{\text {left }}$;

4. Let $x_{1}=x_{2}, y_{1}=y_{2}$ and go to step 2 until $\left(x_{2}, y_{2}\right)$ is no longer located in $\mathbf{S}_{n}$.

5. From the point $\left(x_{0}, y_{0}\right)$, draw the left branch of the predicted crack path by following the same algorithm as above-described. 
For each zone $\mathbf{S}_{n}$, a potential crack path is drawn. Simultaneously, the length of the crack path can be obtained by cumulating $\Delta l$.

\subsection{Creation of cracks by using the maximum energy release rate criterion}

It is clear that even all the predicted crack paths can be drawn by using the above-described algorithm, they will probably not all be activated to become real cracks. Which ones of them become real cracks depend on the fulfillment of the energy criterion $-\delta \Pi / \delta S=G^{i n c} \geq G_{c}$.

All the combinations of the possible crack extensions should be considered in order to find the maximal energy release rate. If we have predicted $N$ potential crack paths and each path may become real crack or not, $2^{N}$ combinations are possible to become real cracks. Consequently, $2^{N}$ linear elastic finite element resolutions have to be performed: one for the structure before crack extension, and $2^{N}-1$ for the structure with all the possible crack extension combinations. The combination which makes $G^{i n c}$ maximum and verifies the Griffith criterion $G^{i n c} \geq G_{c}$ will become real cracks.

For the creation of the new cracks, different approaches exist in the framework of the finite element method. We believe that the algorithm proposed above allows using the following techniques:

1. Remove the elements along the predicted crack paths [25];

2. Remesh the elements near the predicted crack path by creating a new crack [26];

3. Introduce discontinuities along the predicted crack paths [27].

It is clear that amongst all these methods the first one is the simplest. For this reason it was used in the present work. In practice, we find all elements in interception with the predicted crack paths, and then remove them from the finite element model. In return, this obviously requires a sufficiently fine mesh to be as close as possible to the concept of crack.

\subsection{Overall algorithm}

In order to avoid complicated snap-back analyses and to guarantee the existence of the solutions, the external loads are applied incrementally by imposing displacements on the boundaries of the considered structure until a prefixed level. By using the techniques described above, we can construct an overall algorithm as follows:

1. At each loading step, a first finite element calculation is carried out to determine the stress distribution and the total potential energy $\Pi_{0}$ in the structure;

2. With the obtained stress distribution and by using the watershed flooding process, we first find $N$ zones $\mathbf{S}_{n}$ in which $\sigma_{1} \geq \sigma_{c}$ and containing each a local maximum of $\sigma_{1}$;

3. If $N=0$, increase the external loads and go to step 1 .

4. Else, in each such zone $\mathbf{S}_{n}$, a potential crack path is drawn by following a principal stress trajectory; 
5. By activating or not each potential crack path, perform $2^{N}-1$ finite element calculations for all possible combinations and find the corresponding total potential energies $\Pi_{i}$ and the crack length increment $\Delta l_{i}$. For each combination, we calculate the incremental energy release rate $G_{i}^{i n c}=-\frac{\Pi_{i}-\Pi_{0}}{\Delta l_{i}} ;$

6. Consider the crack path having the maximal incremental energy release rate $G_{i}^{\text {inc }}$. If the energy criterion $\max \left(G_{i}^{i n c}\right) \geq G_{c}$ is fulfilled, create the corresponding new cracks by eliminating all elements along the predicted crack paths. Go to step 1 ;

7. Go to step 1 without increasing the external loads.

\section{Validation and numerical examples}

In order to validate the proposed numerical fracture model, we carry out numerical simulations on some special structures for which the fracture modes are well-known or wellstudied in the literature. Through these examples, the efficiency and the accuracy of the model, especially the mesh independency and the capacity in modelling the multiple cracking processes, are also discussed.

\section{(1): Crack initiation from a hole in a plate}

An earlier experimental work [28] showed that the strength of a drilled plate depends closely on the hole size. It was shown that this size effect can be captured by using the coupled criterion. In this work, we will evaluate the remote loads at fracture of the specimens by using the present numerical tool and compare them with the theoretical predictions, obtained by creating an ideal crack from the hole boundary and calculating its exact stress intensity factor according to the CC. Since the material used in the experiments, the polymethyl metacrylate (PMMA), is a very brittle material, the crack initiation leads to immediate structure failure of the specimens. Therefore, the remote load at the crack onset is considered as the strength of the drilled plate.

The mechanical characteristics of the material are as follows: Young's modulus $E=3000$ $\mathrm{MPa}$, Poisson's ratio $v=0.36$; the ultimate tensile stress $\sigma_{c}=72 \mathrm{MPa}$; the critical energy release rate $G_{c}=290 \mathrm{~J} / \mathrm{m}^{2}$. The section of the specimens is of $10 \times 30 \mathrm{~mm}^{2}$. The strengths of the plate with holes of different sizes are evaluated by using the present numerical method. In order to evaluate the influence of the mesh size on the crack onset prediction, three different element sizes near the hole are used in the simulations, namely $d=0.005,0.01$ and $0.02 \mathrm{~mm}$, where $d$ is the average edge length of the triangular elements. It is useful to note that the characteristic length of the material is given by $\left(K_{c} / \sigma_{c}\right)^{2} / 2 \pi=0.026 \mathrm{~mm}$.

The numerical predictions of the strength for different hole sizes are plotted in Figure 2. The ordinate axis represents $\sigma_{\infty} / \sigma_{c}$, the ratio of the remote tensile stress at fracture over the ultimate tensile stress. For comparison, the theoretical predictions obtained by using the $\mathrm{CC}$ $[13,28]$ are also plotted. From this figure, we can remark that the numerical strength 
predictions agree very well with the theoretical ones for fine meshes, i.e., for $d=0.005$ and $0.01 \mathrm{~mm}$. For the coarser mesh $d=0.02 \mathrm{~mm}$, the agreement is still good when the hole diameter is larger than $1 \mathrm{~mm}$. The numerical predictions become slightly more conservative than the theoretical ones when the hole diameter is smaller than $1 \mathrm{~mm}$. As expected, $\sigma_{\infty}$ tends to $\sigma_{c}$ as the hole size tends to zero, it tends to $\sigma_{c} / 3$ as the hole is large.

The main difference between the theoretical and numerical approaches remains in the fact that the creation of ideal cracks in the theoretical approach is replaced by the removed elements in the numerical one. The decrease of the total potential energy is more important in the case when deleting elements, which leads to an over-estimation of the incremental energy release rate. Consequently, the prediction of the crack onset from a non-cracked structure is more conservative by using the element elimination technique. This error can be reduced by using refined mesh. From Figure 2, we can remark that the mesh sizes $d=0.005$ and $0.01 \mathrm{~mm}$ give very satisfactory crack onset predictions comparing to the theoretical predictions. We can also observe that when the mesh is sufficiently refined, the crack onset prediction is very weakly mesh-dependent. It is also worthy to note that the element elimination technique is much simpler to implement in a numerical code than the creation of real cracks, especially for multi-cracking problems.

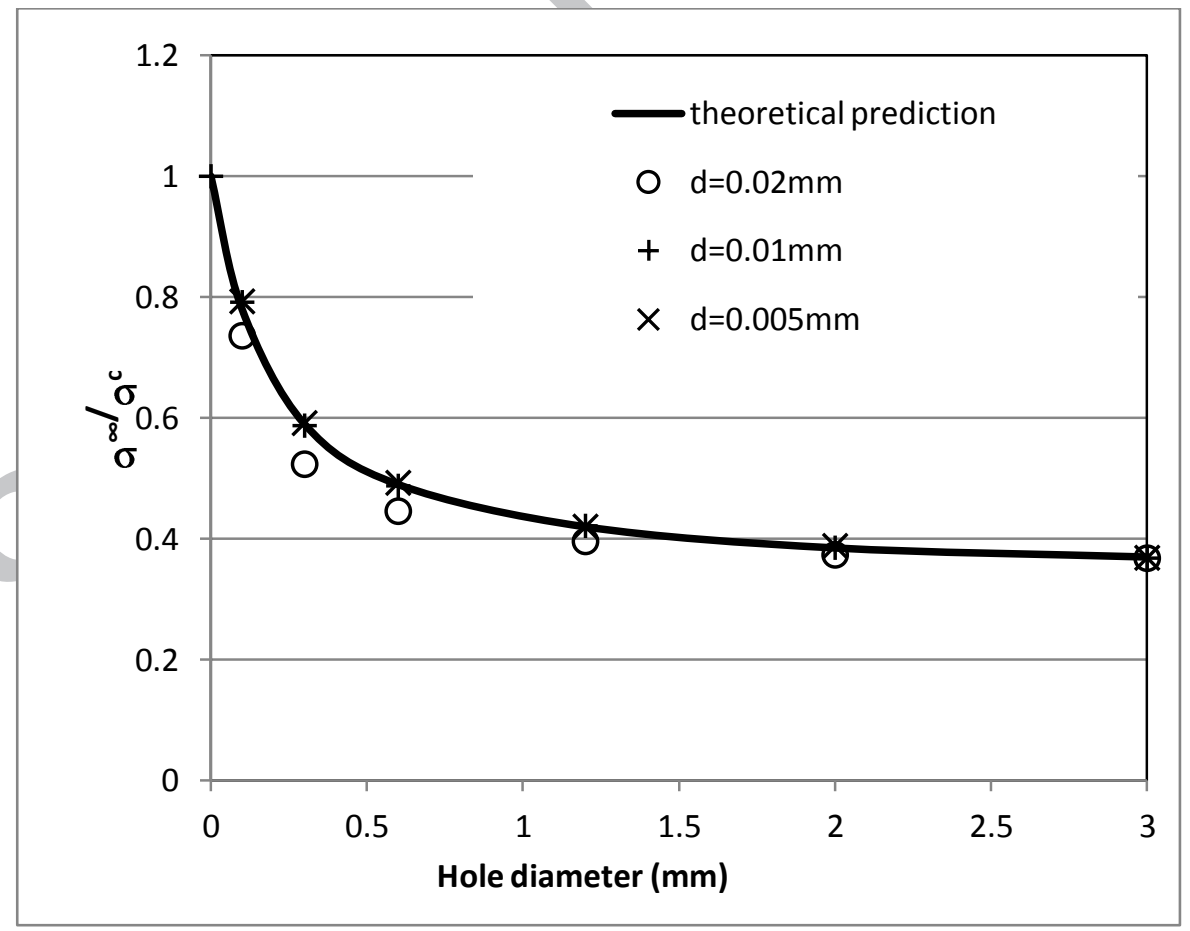

Figure 2: Critical remote stress at fracture of the drilled specimens as function of the hole size. Comparison between the theoretical and numerical predictions

Figure 3 shows the first steps of the crack initiation and growth from the hole boundary. The elements in which the stress condition $\sigma_{1} \geq \sigma_{c}$ is fulfilled are drawn in green. The predicted 
crack path is plotted in black line. The elements drawn in red are at the intersection with the predicted crack path and have to be deleted. The black band represents the already removed elements. The crack onset is predicted according to the $\mathrm{CC}$, i.e., both the stress and energy criteria are fulfilled, that explains the quite lengthy crack initiation (about $0.07 \mathrm{~mm}$, Fig. 3a). After the initiation, the crack grows rapidly under fixed remote displacement loading and it follows unstable crack propagation (Fig. 3b-3c) as observed in the experiments [28].

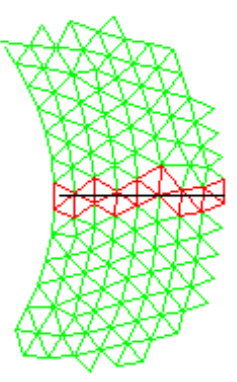

(a)

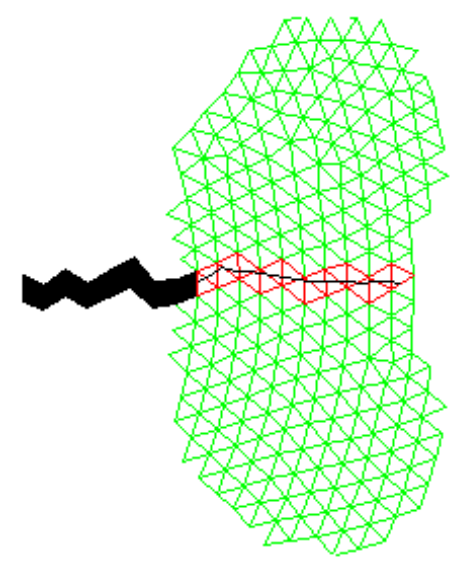

(b)

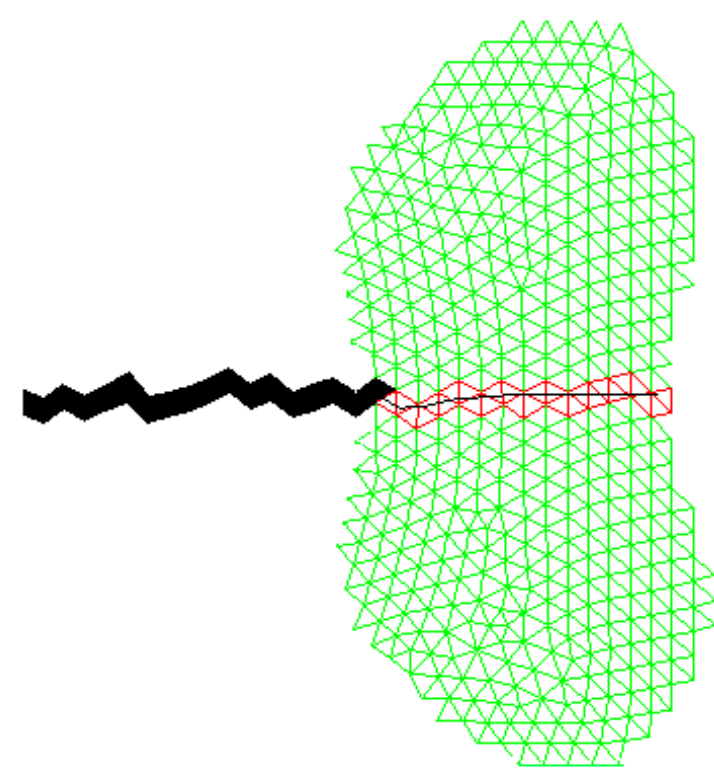

(c)

Figure 3: Numerical simulation of the crack initiation and growth (a): crack onset from the hole boundary (b)-(c): crack growth under unchanged remote displacement load

\section{(2): Crack initiation from the tip of a $V$-notch}

Leguillon [13] performed a detailed study on crack onset from the tip of a sharp V-notch, on the basis of which the $\mathrm{CC}$ was proposed. By using the $\mathrm{CC}$, the remote loads at fracture, the crack direction and the crack onset length were successfully predicted. In fact, the asymptotic stress along the crack path can be written as

$$
\sigma\left(r, \theta_{0}\right)=k r^{\lambda-1} s\left(\theta_{0}\right)
$$

where $\theta_{0}$ is the crack orientation with respect to the $\mathrm{V}$-notch bisector; $r$ is the distance between the $\mathrm{V}$-notch tip and a point at the crack path. The singularity exponent is such that $1 / 2 \leq \lambda \leq 1$ and $k$ is the generalized stress intensity factor. The potential energy change at a crack onset is written:

$$
-\delta \Pi=k^{2} K\left(\theta_{0}\right) l^{2 \lambda} t
$$


where $t$ is the thickness of the specimen; $l$ is the crack onset length and $K$ is a scaling term depending on the opening angle and the crack direction. The use of the CC (i.e. (4) for $0 \leq r \leq$ $l$ and (5)) leads to the crack onset criterion in terms of the generalized stress intensity factor:

$$
k \geq k_{c} \quad \text { with } \quad k_{c}=\left(\frac{G_{c}}{K\left(\theta_{0}\right)}\right)^{1-\lambda}\left(\frac{\sigma_{c}}{s\left(\theta_{0}\right)}\right)^{2 \lambda-1}
$$

In [13], the coefficients $k$ and $K$ were evaluated by means of the matched asymptotic procedure. The crack direction $\theta_{0}$ maximizes the denominator in the right hand side of (6).

Hereafter we evaluate the remote loads at crack onset of a V-notched specimen and compare the results with Leguillon's predictions. The geometry and the loading condition of the specimen are illustrated in Figure 4. The thickness of the specimen is $e=5 \mathrm{~mm}$. Displacements are prescribed at its top and bottom ends. The material parameters are as follows: Young's modulus $E=3000 \mathrm{MPa}$, Poisson's ratio $v=0.36$; the ultimate tensile stress $\sigma_{c}=72 \mathrm{MPa}$; the critical energy release rate $G_{c}=290 \mathrm{~J} / \mathrm{m}^{2}$. The $\mathrm{V}$-notch opening angle is $2 \beta$ $=90^{\circ}$. Fine mesh is used around the V-notch tips with the element size $d=0.005 \mathrm{~mm}$. Three loading cases, the pure tension, the pure shear and a mixed mode, are studied (Table 1). Eqn. (6) can be used in the two first cases, considering either the first (symmetric) or second (antisymmetric) mode of the Williams expansion associated with two different exponents $\lambda$ (Table 1). In the more entangled mixed mode, an extension of (6) must be used, taking into account together the two above mentioned modes. See [29,30] for the generalization of (6) to complex loadings.

The remote prescribed displacements $u_{1}$ or $u_{2}$ are incrementally increased until the CC is fulfilled. The critical remote resulting forces $F_{1}$ for shear and $F_{2}$ for tension obtained in this way are compared with the results using Leguillon's method, as shown in the following table:

Table 1: Critical remote loads at crack initiation of V-notched specimens

\begin{tabular}{|l|l|l|l|}
\hline $\begin{array}{l}\text { Prescribed } \\
\text { displacements }\end{array}$ & $\begin{array}{l}u_{1}=0 \text { and } u_{2} \neq 0 \\
\text { pure tension }\end{array}$ & $\begin{array}{l}u_{1} \neq 0 \text { and } u_{2}=0 \\
\text { pure shear }\end{array}$ & $\begin{array}{l}u_{1} / u_{2}=10 \\
\text { mixed mode }\end{array}$ \\
\hline Leguillon's method & $\lambda=0.545$ & $\lambda=0.906$ & $\lambda_{1}=0.545, \lambda_{2}=0.906$ \\
& $\theta_{0}=0 \mathrm{deg}$. & $\theta_{0}=57 \mathrm{deg}$. & $\theta_{0}=35 \mathrm{deg}$. \\
& $F_{1}=0$ & $F_{1}=10819 \mathrm{~N}$ & $F_{1}=4731 \mathrm{~N}$ \\
& $F_{2}=2048 \mathrm{~N}$ & $F_{2}=0$ & $F_{2}=1752 \mathrm{~N}$ \\
\hline $\begin{array}{l}\text { Present numerical } \\
\text { method }\end{array}$ & $\theta_{0}=0 \mathrm{deg}$. & $\theta_{0}=54 \mathrm{deg}$. & $\theta_{0}=31 \mathrm{deg}$. \\
& $F_{1}=0$ & $F_{1}=10931 \mathrm{~N}$ & $F_{1}=4611 \mathrm{~N}$ \\
& $F_{2}=2082 \mathrm{~N}$ & $F_{2}=0$ & $F_{2}=1746 \mathrm{~N}$ \\
\hline
\end{tabular}

This comparison shows that the numerical predictions agree very well with the matched asymptotic procedure proposed in [13]. The differences on the critical remote loads are limited within $2 \%$. The agreement between the crack directions may seem less accurate but it 
must be pointed out that it is difficult to measure in the numerical simulation, where the crack has a somewhat saw-tooth appearance caused by the removed elements.

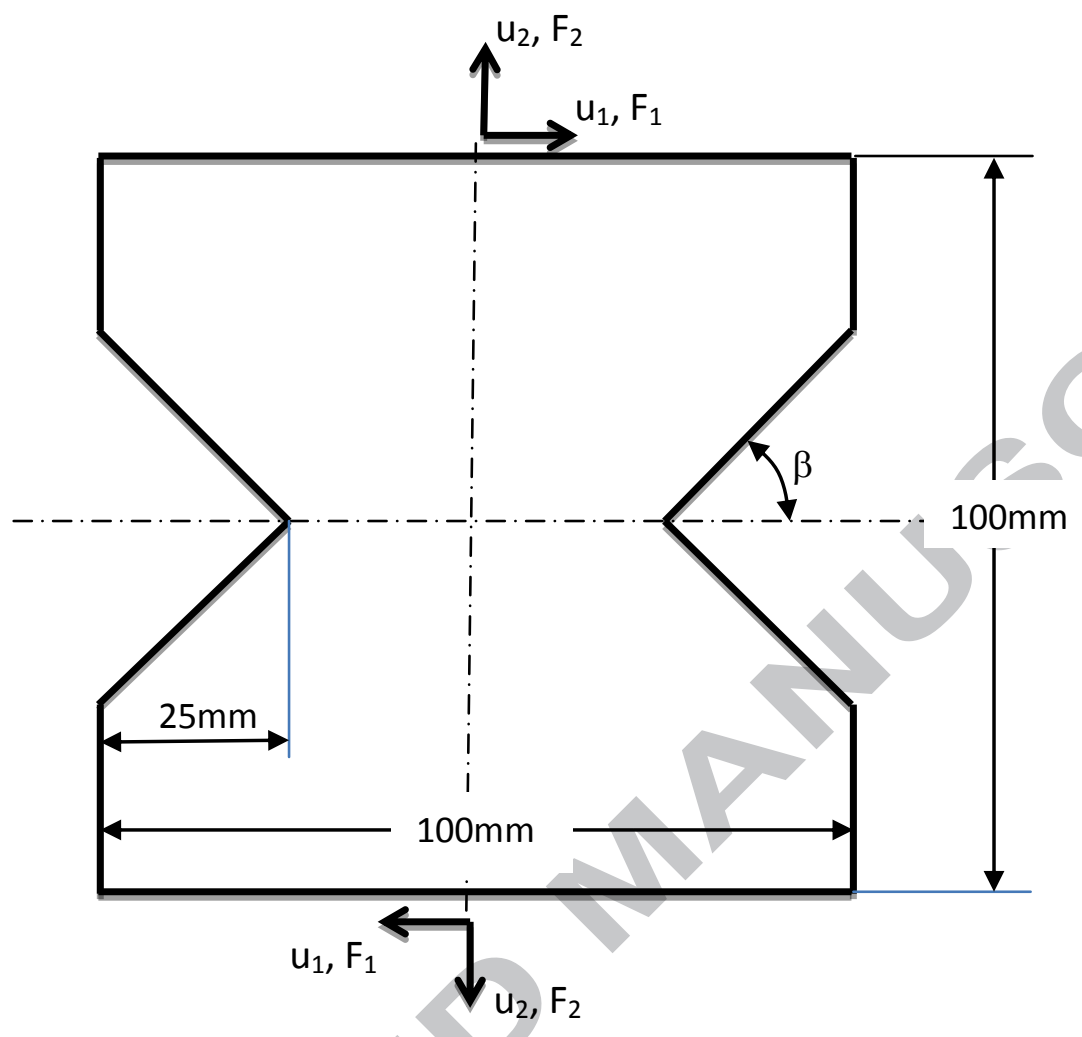

Figure 4: Geometry and load conditions of the V-notched specimen

\section{(3): Crack initiation and growth}

The third example in the validation of the present model is a plate including a central crack subjected to a pure mode-I remote loading. We will show that in this case, the CC is equivalent to the Griffith criterion if the pre-existing crack is long enough.

Consider a 2D square plate with a central crack of length $2 a$. The dimension of the specimen is $2 W \times 2 W=10 \times 10 \mathrm{~mm}^{2}$. We evaluate the critical remote loads for crack growth with a central crack of different sizes, namely $a / W=0,0.02,0.04,0.06,0.08,0.1,0.2,0.3,0.4$ and 0.5 , here $a=0$ means that there is no crack in the plate. The mechanical properties of the material are: Young's modulus $E=3000 \mathrm{MPa}$, Poisson's ratio $v=0.3$, ultimate stress $\sigma_{c}=$ $150 \mathrm{MPa}$, critical stress intensity factor $K_{c}=67 \mathrm{MPa} \sqrt{\mathrm{mm}}$. By considering the symmetry, only one half of the plate is modeled. In order to investigate the influence of the mesh size, different element sizes near the crack tip are used in the simulations, namely $d=0.003,0.006$ and $0.01 \mathrm{~mm}$, where $d$ is the average edge length of the triangular elements. A uniaxial tension along the direction normal to the crack was applied at the ends of the plate by imposing incrementally increasing displacements. The remote load is applied gradually until the CC is verified. Figure 5 shows the fracture details near the crack tip. 


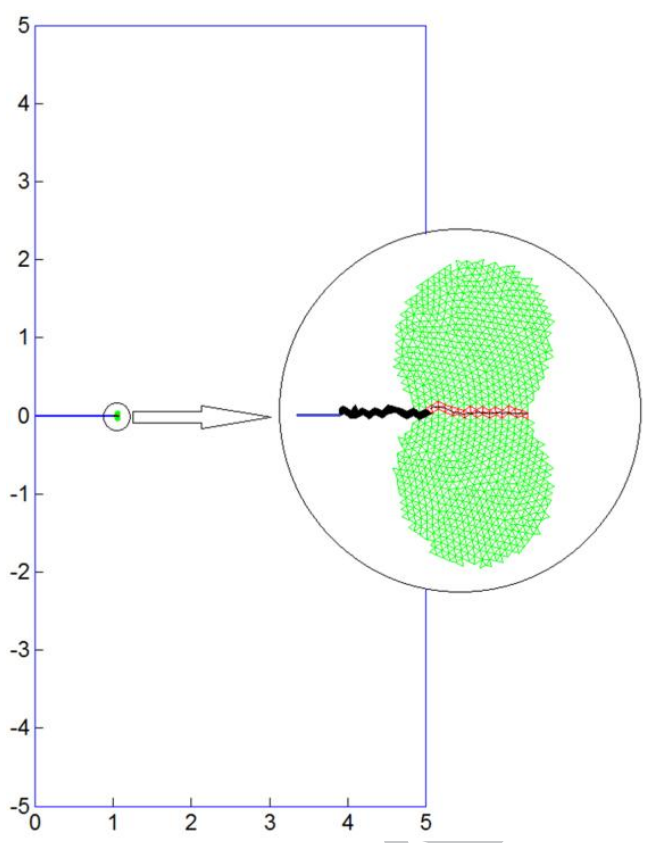

Figure 5: Growth simulation of a central crack. The black band represents the removed elements after the first step of the crack growth.

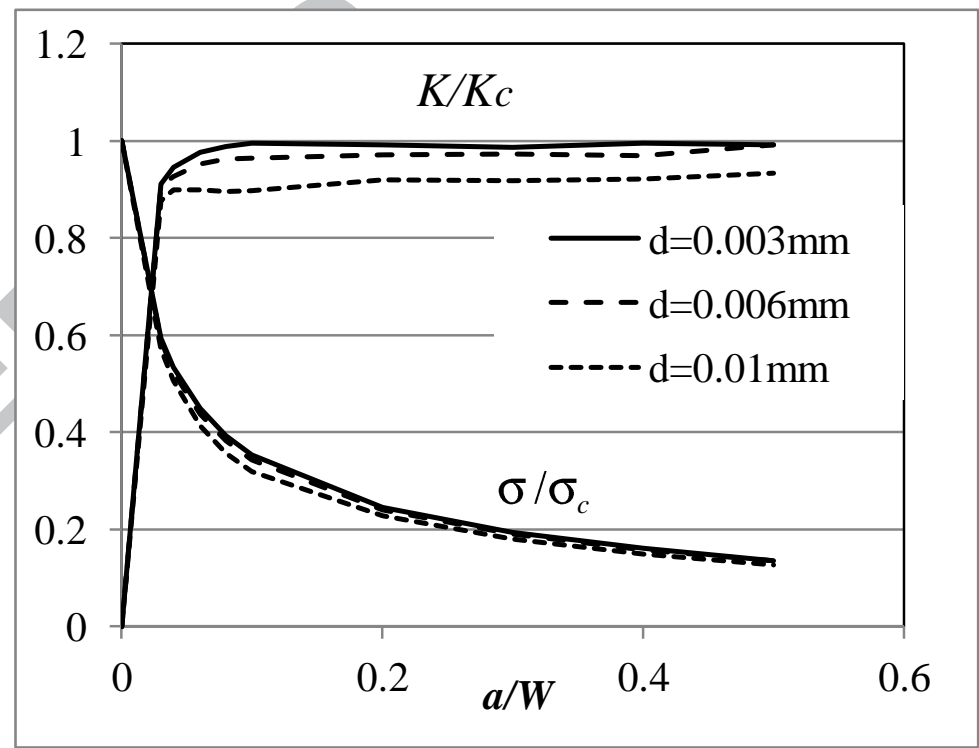

Figure 6: Normalized stress intensity factor $K_{\mathrm{I}} / K_{\mathrm{Ic}}$ and normalized remote stresses $\sigma^{\infty} / \sigma_{c}$ as a function of the normalized semi-crack length $a / W$ for mode-I loaded cracks at fracture.

The critical remote load at crack propagation thus obtained was used to calculate the stress intensity factor by using a formula found from a handbook of stress intensity factors [31]. By comparing the obtained stress intensity factors to the critical value $K_{c}$, we can evaluate the accuracy of the method for crack growth prediction. The results of these calculations are 
shown in Figure 6. From this figure, we can observe that for sufficiently long cracks $(a / W>$ $0.05)$, the normalized stress intensity factor $K / K_{c} \approx 1$. Therefore, the stress intensity factors at fracture evaluated by using the $\mathrm{CC}$ equal correctly the material fracture toughness. For very short cracks, the Griffith fracture criterion is no longer sufficient to predict the crack growth. The critical stress intensity factor decreases as the crack length decreases. Moreover, the remote stress at fracture tends to the material tensile strength as the crack length tends to zero. In this case, the $\mathrm{CC}$ degenerates to the maximum principal stress criterion for nonsingular stresses.

We can observe that the crack growth prediction is very weakly mesh-sensitive when sufficiently refined meshes are used. Apart from the results calculated with $d=0.01 \mathrm{~mm}$ that represents quite a coarse mesh, the calculations with finer meshes, e.g. $d=0.003 \mathrm{~mm}$ and $0.006 \mathrm{~mm}$, provide us with very close values for remote loads at fracture. In fact, the predicted crack path should contain sufficient number of elements in order to guarantee a good accuracy on the stress and energy calculations. For crack growth, the length of predicted crack path can be calculated by $l=\left(K_{c} / \sigma_{c}\right)^{2} / 2 \pi \approx 0.032 \mathrm{~mm}$ for the used material with the parameters above-mentioned. From Figure 6 , we remark that the element size $d=l / 5$ offers already sufficient accuracy for the crack growth prediction. The complete mesh-independency can be expected by using very refined meshes, which will increase drastically the computational costs.

\section{(4): Crack path tracking: interaction of two cracks}

The present method enables efficient crack path tracking when the stress field is accurately evaluated. We illustrate this feature by the following example. Consider a rectangular plate with two non-symmetric cracks at its side edges subjected to tensile loading (Figure 7). We calculate the critical remote load at fracture and the crack paths by using the proposed method and then compare them with the results found in the literature. Recently, Judt and Ricoeur [32] studied this problem by using a variant of the $J$-integral technique [33]. The geometry of the specimen is shown in Figure 7. The material parameters are chosen as follows: Young's modulus $E=72000 \mathrm{MPa}$, Poisson's ratio $v=0.33$, ultimate stress $\sigma_{c}=300 \mathrm{MPa}$ and material fracture toughness $K_{c}=23.9 \mathrm{MPa} \sqrt{\mathrm{m}}$.

It is clear that the initial geometrical symmetry of the crack positions is unstable. From experimental or numerical point of view, a small perturbation can break this symmetry such that one of the cracks can first grow while the other remains arrested. In order to keep approximately the geometrical symmetry during the crack propagation, we enforce the simultaneous growth of the two cracks, even though the minimum potential principle may be slightly violated.

In order to assess the mesh-sensitivity of the method, we performed simulations with 3 element sizes, namely $d=0.075 \mathrm{~mm}, 0.1 \mathrm{~mm}$ and $0.15 \mathrm{~mm}$. In Figure 7 , we show the crack paths obtained with the different meshes. For comparison, the crack paths obtained in [32] are 
also plotted in this figure. We can see that the three meshes provide almost the same crack paths which agree very well with the result presented in [32]. Here again, the mesh-sensitivity is shown very weak with the adopted meshes and the accuracy of the proposed model is satisfactory in predicting the crack paths.

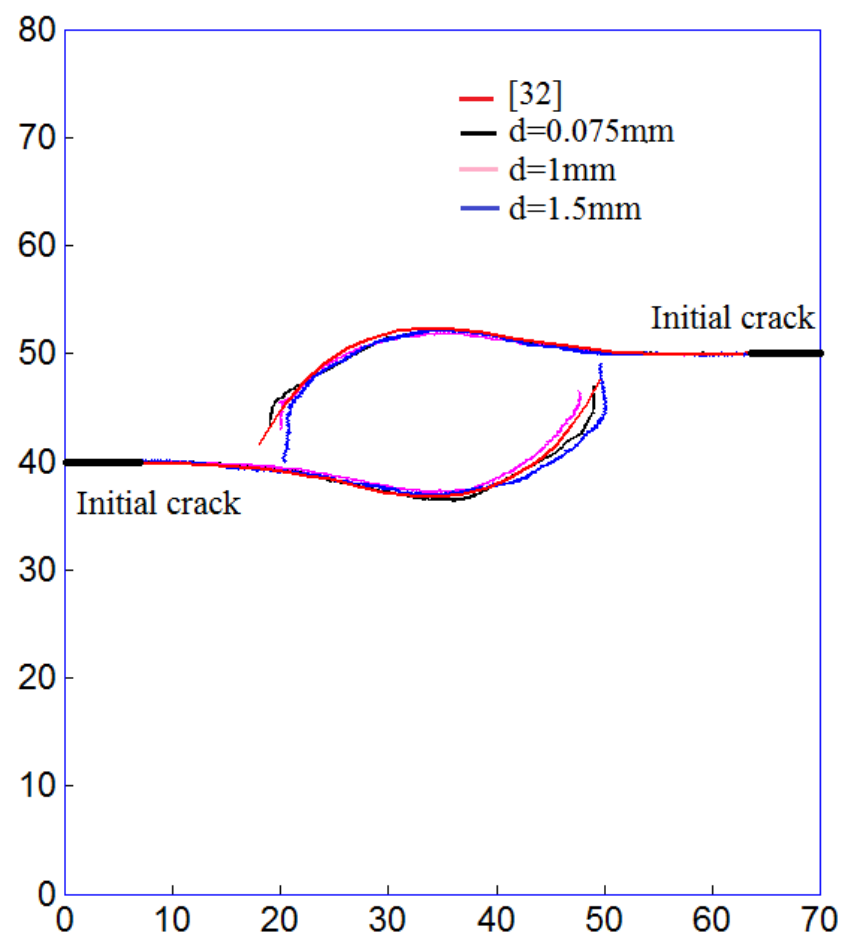

Figure 7: Crack paths predicted by using different meshes in a plate with double cracks

\section{(5): Multi-cracking: Cracking in fiber-reinforced epoxy cross-ply laminates}

Multiple crack initiations and growths can occur simultaneously. Simulation of multiple cracking processes is a big challenge for computational mechanics. The proposed numerical model is able to deal with this problem in a rigorous manner. In fact, all the possible new crack loci are examined by applying the $\mathrm{CC}$ and the best combination of them that makes the total potential energy minimal is selected as the next cracks extensions.

In the following, we consider the experimental works performed by Parvizi et al. [34] on the transverse fracture of glass fiber-reinforced cross-ply laminates. The numerical simulations of their experiments can be used to validate Leguillon's criterion and also, to show the ability of the proposed method in modelling the multi-cracking process.

Parvizi et al. [34] carried out tensile tests on a cross-ply glass fiber-reinforced composite made of two outer layers oriented at $0 \circ$ (fibers are parallel to the tension direction) and an inner one oriented at $90^{\circ}$, as shown in Figure 8 . The thickness of the inner ply was varied and the applied strain at the first occurrence of a transverse crack was recorded. As the applied load increases, multiple cracks were observed in the inner ply. 


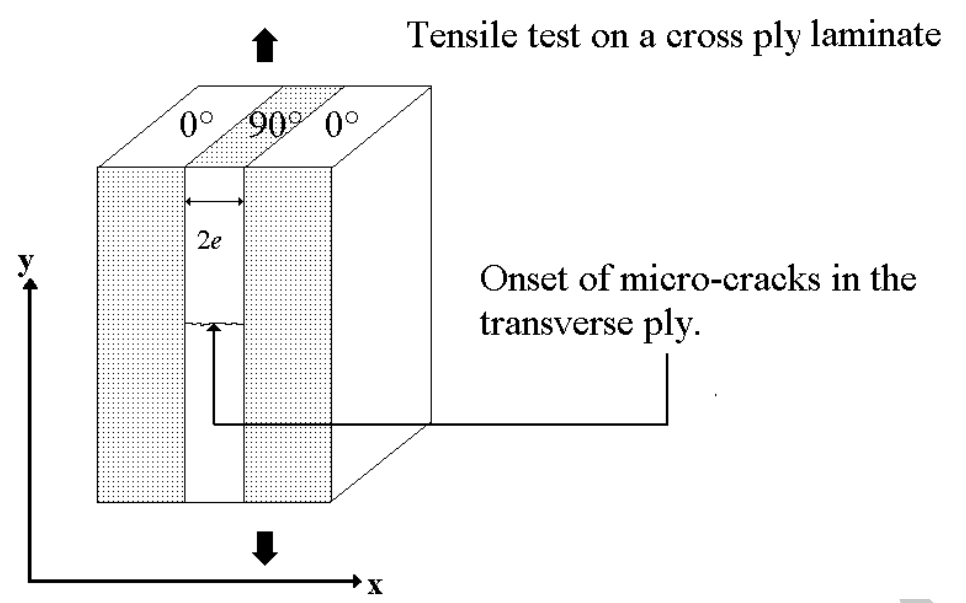

Figure 8: Scheme of the tensile tests on a cross-ply glass fiber-reinforced composite Include the

The samples were modeled by using bi-dimensional plane-strain triangle elements. Only one half of the sample was meshed by symmetry. The plies were regarded as orthotropic materials with the following Hooke's law:

$$
\left\{\begin{array}{l}
\sigma_{x x} \\
\sigma_{y y} \\
\sigma_{x y}
\end{array}\right\}=\left[\begin{array}{ccc}
\frac{E_{x}}{1-v_{x}^{2}} & \frac{v_{x} E_{x}}{1-v_{x}^{2}} & 0 \\
\frac{v_{x} E_{x}}{1-v_{x}^{2}} & \frac{E_{y}}{1-v_{y}^{2}} & 0 \\
0 & 0 & \mu_{x y}
\end{array}\right]\left\{\begin{array}{c}
\varepsilon_{x x} \\
\varepsilon_{y y} \\
\gamma_{x y}
\end{array}\right\}
$$

According to the data given in [32], the mechanical properties of the outer ply are: $E_{x}=14000$ $\mathrm{MPa} ; E_{y}=42000 \mathrm{MPa} ; v_{x}=0.13 ; v_{y}=0.4 ; \mu_{x y}=10000 \mathrm{MPa}$, and for the inner ply : $E_{x}=E_{y}=$ $14000 \mathrm{MPa} ; v_{x}=v_{y}=0.13 ; \mu_{x y}=6194 \mathrm{MPa}$. The outer ply was considered as unbreakable. For the inner ply, according to [34], we took $\sigma_{c}=84 \mathrm{MPa}$ and $G_{c}=0.12 \mathrm{MPa} \mathrm{mm}$. The length of the samples is taken to be sufficiently long $(L=10 \mathrm{~mm})$ such that the appearance of the first crack is not influenced by the length of the samples. The thickness of the outer ply is $0.5 \mathrm{~mm}$. Several thicknesses of the inner ply were considered in the simulation, namely $2 e=0.05,0.1$, $0.2,0.3,0.4,0.5,1,4 \mathrm{~mm}$ respectively. The specimens were subjected to uniaxial tension in the vertical direction by imposing incrementally increasing displacements on the outer layers of the composite.

We first illustrate how the proposed method initiates the first crack in the inner ply. Figure 9-a shows the distribution of the first principal stresses in the inner ply just before the onset of the first crack. In Figure 9-b, we plot the first principal stresses of the inner ply along the interface. From these two figures, we can find that the stress concentrations are of a very high level at the two ends of the specimen near the interface. At the center of the specimen, it appears a very large zone in which the first principal stress exceeds the material strength with a nearly uniform distribution. The proposed algorithm should choose the location of the first 
crack initiation according to the CC. In fact, three zones including a local stress maximum were first found by applying the watershed line method, as shown in Figure 9-c. Then the potential crack paths were created from the local stress maxima by following the direction normal to the first principal stress. As shown in Figure 9-d, although the stress concentration is much higher at extremities than in the central part of the specimen, the potential crack paths are much shorter. Consequently, these potential cracks have small impact on the total potential energy so that the energy criterion is not fulfilled. On the contrary, the potential crack at the central part of the specimen is much longer. Its activation provokes a much larger variation of the total potential energy. Consequently, both the stress and energy criteria are fulfilled to create first a new crack at the central part of the specimen. This is what was observed in the experiments [34].

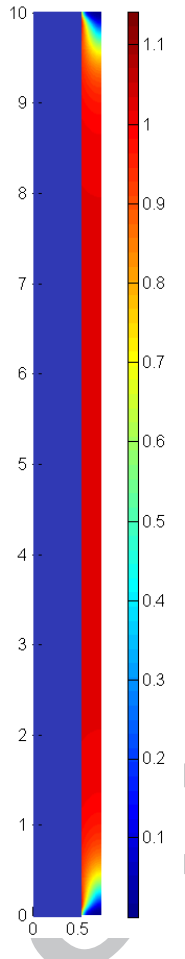

(a)

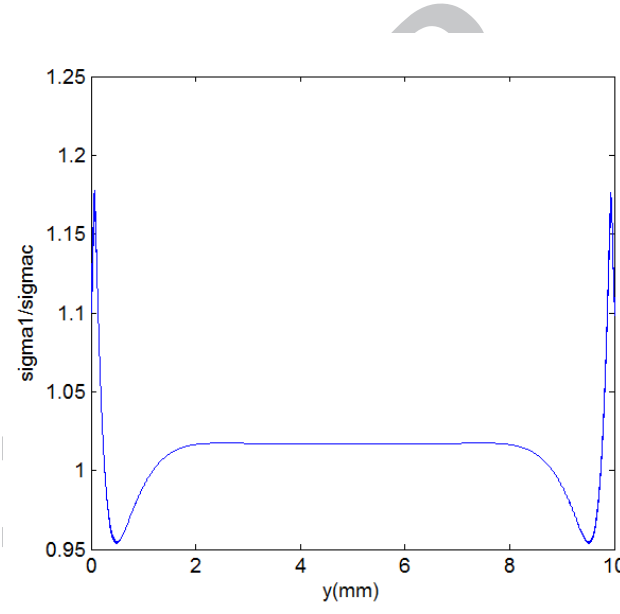

(b)

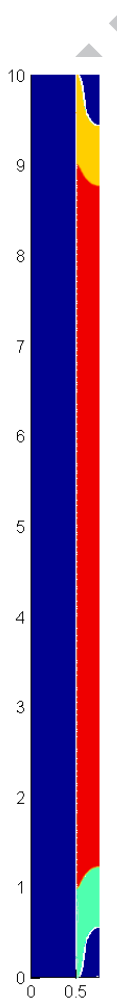

(c)

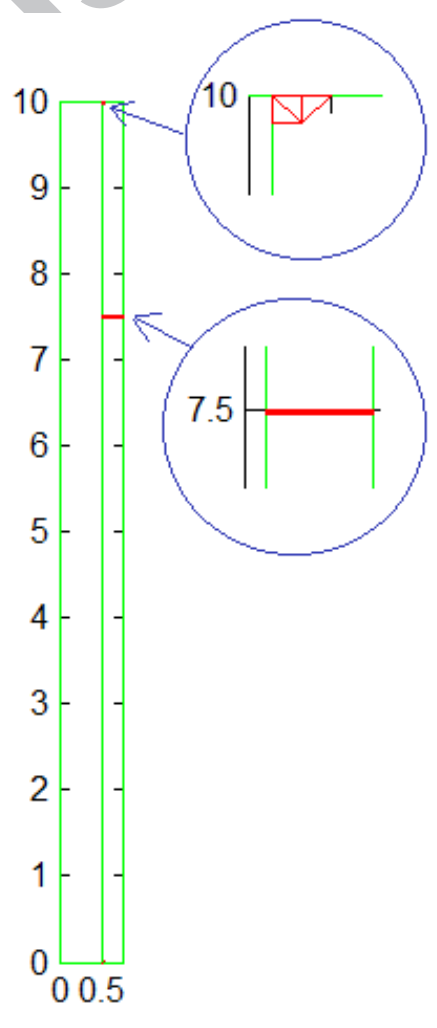

(d)

Figure 9: Stress distribution and the potential crack paths. (a) Distribution of $\sigma_{1} / \sigma_{c}$; (b) Variation of $\sigma_{1} / \sigma_{c}$ along the interface; (c) Three zones found in which there is a local maximum of $\sigma_{1} / \sigma_{c} \geq 1$; (d) Potential crack paths

By following this method, we simulated the experiments for specimens of different thicknesses of the inner layer. The imposed longitudinal strains at first crack initiation were obtained, then compared with the experimental data of Parvizi et al., as shown in Figure 10. We can remark a very satisfactory agreement between the numerical and experimental results. 
In fact, for specimens with a thin inner ply, the energy criterion governs the failure while the stress criterion is trivially fulfilled. The contrary occurs for specimens with a thick inner ply.

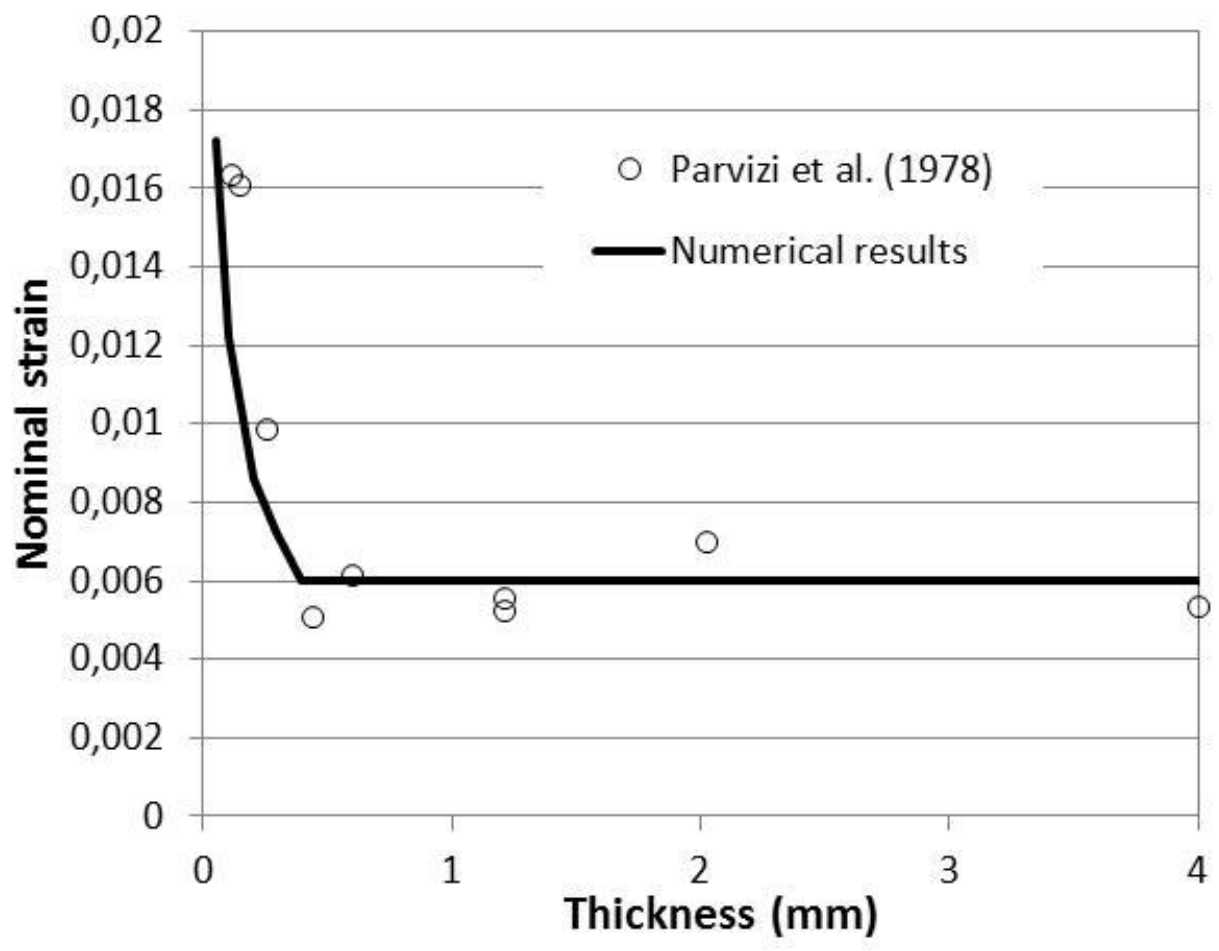

Figure 10: Nominal strain at the occurrence of the first transverse crack versus the thickness of the inner ply

It is clear that one of the interesting features of the proposed method is its ability to simulate the multi-cracking process in composite materials for instance. The further crack creation process in the laminate was simulated for different thicknesses of the inner ply. In Figure 11, we plot the density of the transverse cracks in the inner ply as a function of the prescribed strain. As expected, specimens with a thin inner ply sustain higher loads for the first crack formation. However, the crack density increases rapidly as the load increases. For specimens with a thick inner ply, the first crack formation requires smaller remote loads. As the stress relaxation is larger when creating a longer crack, the further crack formation requires higher remote loads. This trend was also observed in some experimental works [35] on thin coatings. 


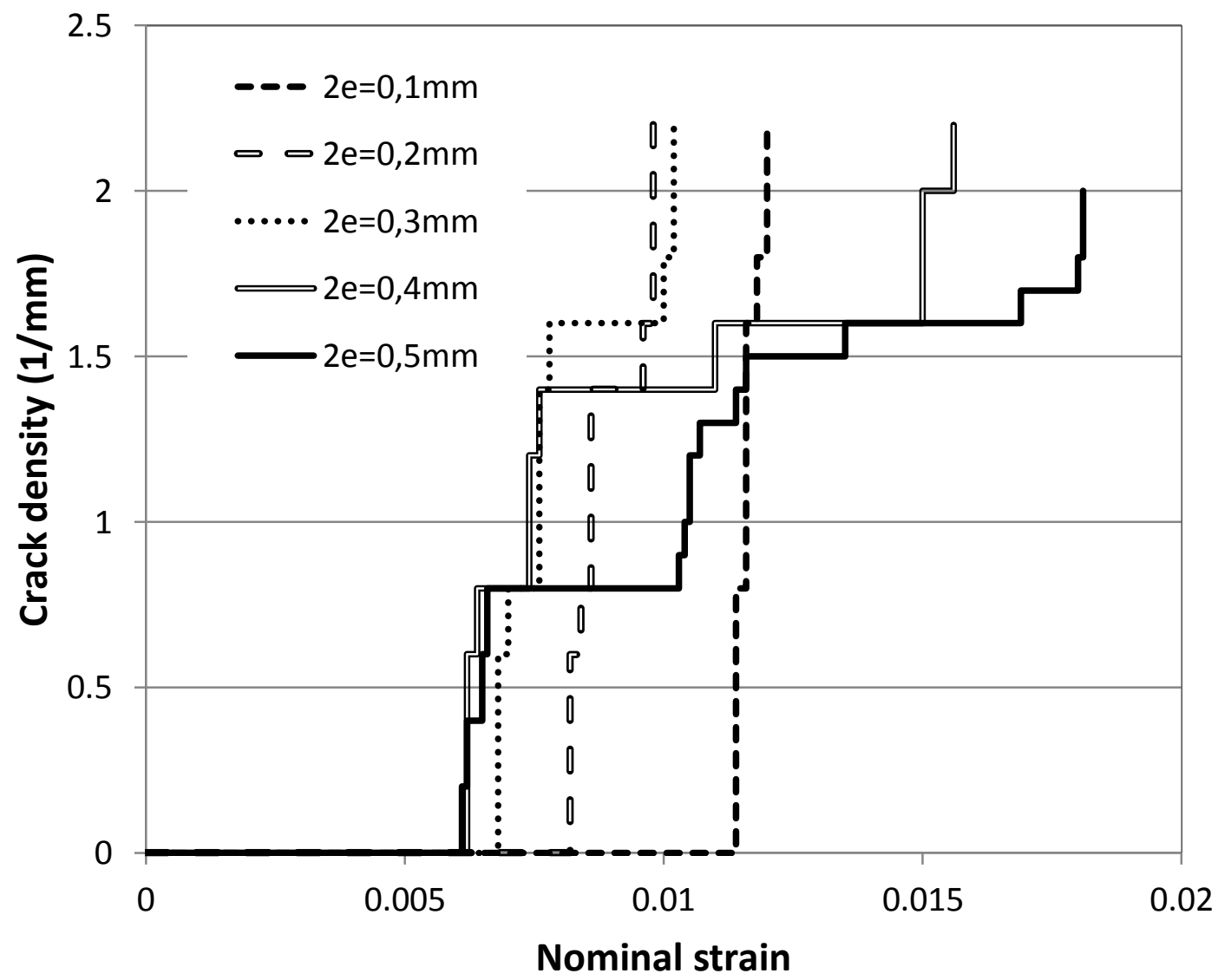

Figure 11: Crack density as function of Nominal strain for different thickness of the inner ply

\section{(6): Cracking in composite materials}

In this section, we attempt to assess the multi-cracking process in composite materials. In this case, the strengths and the fracture toughness are function of the spatial coordinates. In this case, the watershed technique permits to create automatically distinct potential cracks in adjacent materials of the composite. According to the CC, we have to ensure that $-\delta \Pi / \delta S$ is maximal for the crack combination. However, a global energy criterion $-\delta \Pi / \delta S \geq G_{c}$ does not exist for creating several cracks simultaneously as the values of $G_{c}$ may be different for each crack. In order to overcome this difficulty, we assume furthermore that the energy criterion $-\delta \Pi^{(i)} / \delta S^{(i)} \geq G_{c}^{(i)}$ is fulfilled for all the individual cracks of the combination, where $\delta \Pi^{(i)}, \delta S^{(i)}, G_{c}^{(i)}$ are respectively potential energy change, the newly created crack surface and the critical energy release rate when only the crack $(i)$ is activated in a single material component of the composite.

To illustrate this feature, we consider again the fiber-reinforced laminate formerly treated in example (5) by taking some scattering into account. In the following simulations, we assume that the strength and the fracture toughness are randomly distributed in the inner ply of the 
composite following a Weibull distribution. The two parameters Weibull's law used in this work writes:

$$
f\left(\sigma_{c}\right)=\frac{m}{\sigma}\left(\frac{\sigma_{c}}{\sigma}\right)^{m-1} e^{-\left(\frac{\sigma_{c}}{\sigma}\right)^{m}}
$$

where $f\left(\sigma_{c}\right)$ stands for the failure probability at $\sigma_{1}=\sigma_{c}, m$ and $\sigma$ are the Weibull parameters, with $m=9$ and $\sigma=89 \mathrm{MPa}$. Following this probability function, the material strength is randomly distributed in the inner ply by spots of $0.5 \mathrm{~mm} \times e$. The fracture toughness of each spot is related to the strength by $G_{c}=0.132\left(\sigma_{c} / \sigma\right)^{2} \mathrm{MPa}$.mm. These strength and fracture toughness distributions will generate approximately a mean strength of $84 \mathrm{MPa}$ and a mean fracture toughness of $0.12 \mathrm{MPa} . \mathrm{mm}$ as those in example (5). One of the strength distributions and the frequency histogram are shown in Figure 12.

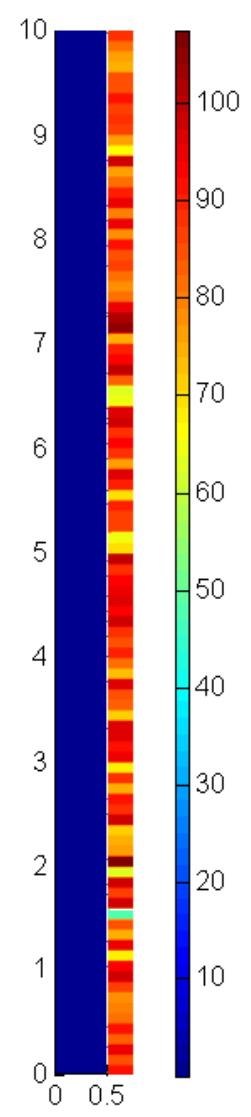

(a)

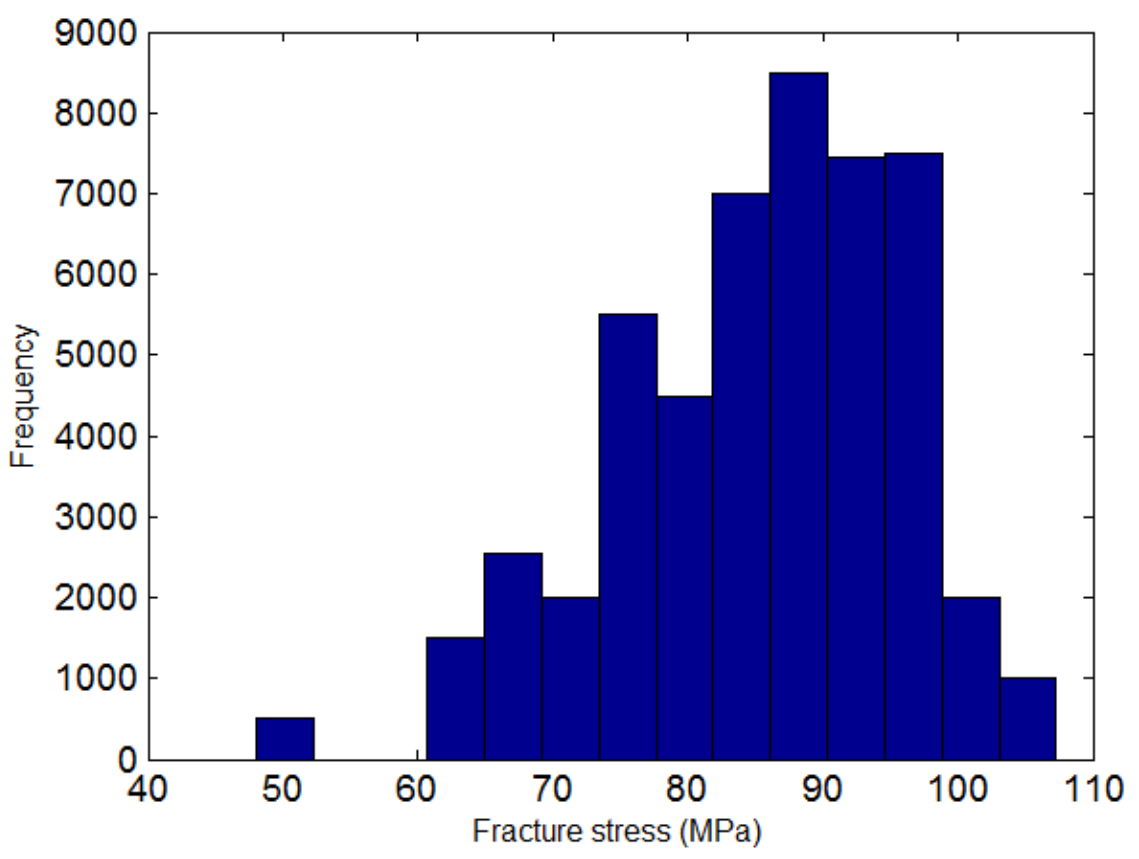

(b)

Figure 12: (a) Strength distribution in the inner ply according to the Weibull model (b)

Histogram of strength

By means of this probabilistic approach, the previous numerical simulations were revisited. The numerical results are plotted in Figure 13. Comparing to Figure 11, the curves of crackdensity vs. nominal strain show more graduate variation with a gentle slope. These results exhibit a better similarity to those obtained in experiments [35]. 
Figure 14 shows the crack patterns in the inner ply obtained by using the deterministic and probabilistic approaches respectively. Noting that the crack pattern of the deterministic model is very regular; the cracks appear by following nearly a dichotomy rule, while the probabilistic model provides a more irregular crack pattern. We can also note that small cracks emerge when the crack density reaches a critical level for both the deterministic and probabilistic models. In real composite materials, the interface debonding is often observed instead of the small transverse cracks. As the interface debonding is not taken into account in the present work, the small transverse cracks appear in the numerical simulations.

This example shows numerous capacities of the proposed method in simulating multicracking process in heterogeneous materials. As our attention is focused on the methodology description, detailed comparison with experimental works has not been realized.

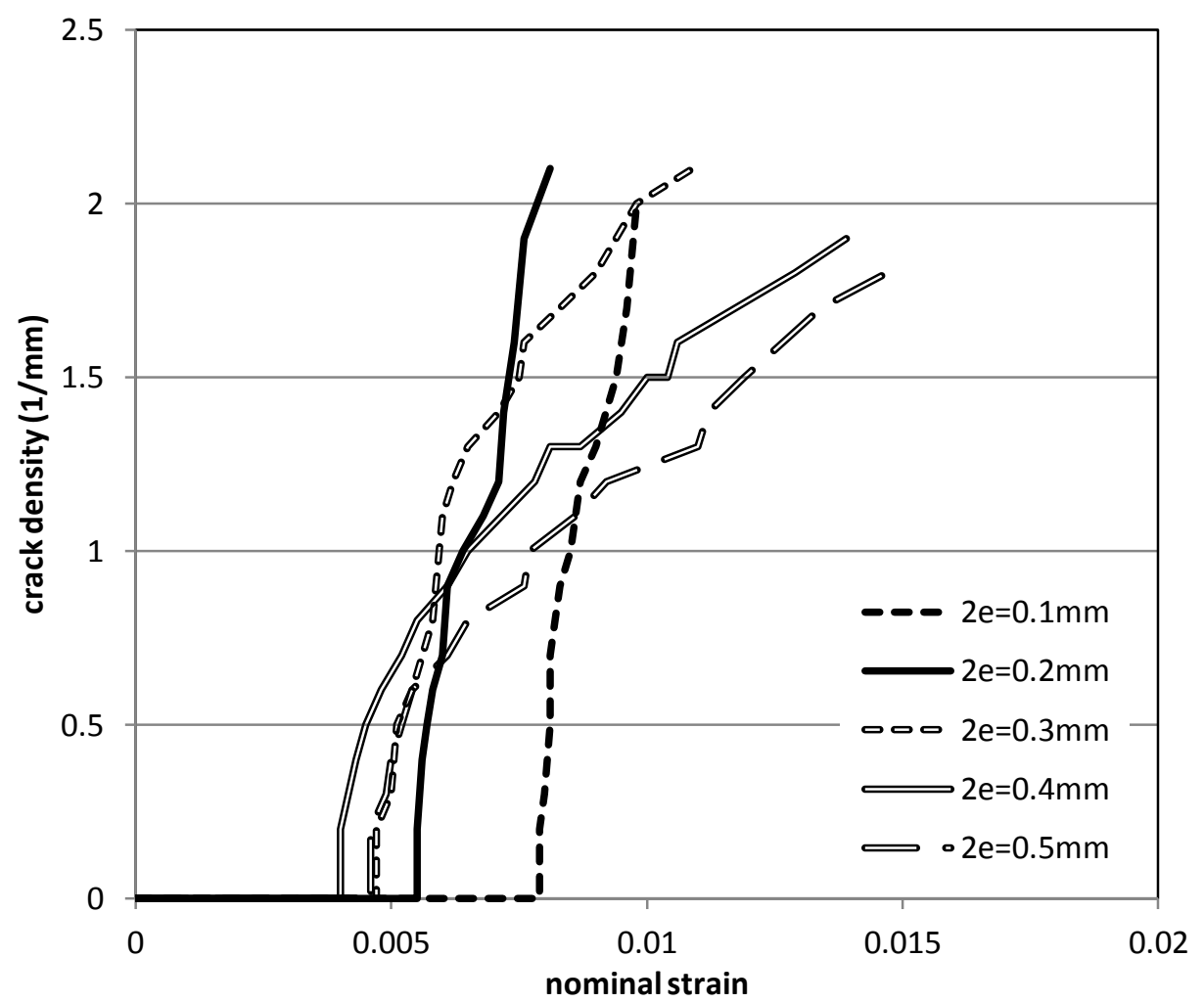

Figure 13 Crack density as function of Nominal strain for different thickness of the inner ply according to the Weibull model 

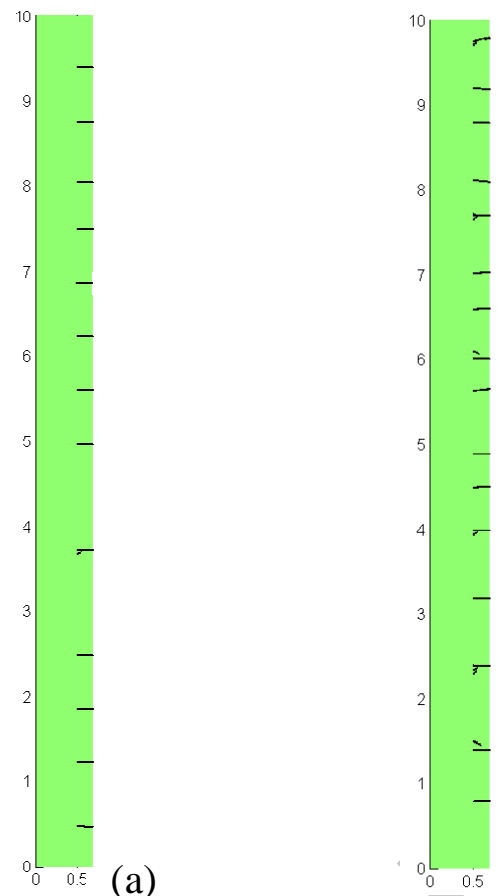

(b)

Figure 14: Crack patterns in the inner ply obtained by using the deterministic (a) and probabilistic (b) approaches respectively at nominal strain $\varepsilon=0.0115$

\section{Conclusions}

In this work, we have implemented the fracture criterion proposed by Leguillon [13] into a finite element code for simulating two-dimensional cracking process in brittle materials. By means of a large number of examples for which the solutions are well-known or well-studied in the literature, the validity of the proposed numerical model was confirmed. It was shown that this numerical model presents several advantages, for example:

- Both crack initiation and crack growth can be predicted;

- Multi-cracking problems can easily be considered;

- Cracking process in heterogeneous materials can be simulated;

- Once the finite element model provides sufficient accuracy on stress evaluation, the fracture prediction is nearly mesh-insensitive.

- Convergence can always be guaranteed if displacement boundary conditions are prescribed.

- Only linear elastic calculations are required. This allows building a robust and efficient computational algorithm.

It is clear that further researches are necessary to improve the numerical model. One of the tasks is to build smooth stress fields in order to avoid the over-segmentation problem that leads to too many potential cracks. In this topic, the denoising techniques developed in image processing science [24] should be more thoroughly investigated. Another drawback of the model may be its huge computational costs required for a large number of cracks. Even though parallel computing techniques can largely increase the calculation speed, 
supplementary hypotheses and more efficient algorithms are expected to reduce the computational tasks.

The couple criterion for fracture prediction is conceptually simple and physically reasonable. Its numerical implementation opens a new way to describe the cracking process in solids. We believe that many other complex problems in fracture evaluations can be treated by developing more operational numerical tools.

\section{References}

1. G.I. Barenblatt, The mathematical theory of equilibrium cracks in brittle fracture, Adv. Appl. Mech. 7 (1962) 55-129.

2. D. Dugdale, Yielding of steel sheets containing slits, J. Mech.Phys.Solids, 8(2) (1960) 100104.

3. X.P. Xu, A. Needleman, Numerical Simulation of Fast Crack Growth in Brittle Solids. J. Mech. Phys. Solids 42 (1994) 1397-1434.

4. G.T. Camacho, M. Ortiz, Computational modelling of impact damage in brittles materials, Int. J. Solids Struct. 33 (1996) 2899-2938.

5. A. Needleman, V. Tvergaard, Finite element analysis of localization in plasticity. In: J.T. Oden and G.F. Carey (Eds.), Finite elements, Special problems in solid mechanics, PrenticeHall, New Jersey, 1984 pp. 94-157.

6. A. Needleman, Some issues in cohesive surface modeling, 23rd International Congress of Theoretical and Applied Mechanics, Procedia IUTAM 10 ( 2014 ) 221-246.

7. G. Francfort, J.J. Marigo, Revisiting brittle fracture as an energy minimization problem. J. Mech. Phys.Solids 46 (1998) 1319-1342.

8. B. Bourdin, G. Francfort, J.J Marigo, Numerical experiments in revisited brittle fracture. J. Mech. Phys. Solids 48 (2000) 797-826.

9. H. Amor, J.J. Marigo, C. Maurini , Regularized formulation of the variational brittle fracture with unilateral contact: numerical experiments. J. Mech. Phys.Solids 57 (2009)1209-1229.

10. J. Vignollet, S, May, R. de Borst, C.V. Verhoosel, Phase-field models for brittle and cohesive fracture. Meccanica 49 (2014) 2587-2601.

11. S. May, J. Vignollet, R. de Borst, A numerical assessment of phase-field models for brittle and cohesive fracture: G-Convergence and stress oscillations, Europ. J. Mech. A/Solids 52 (2015) 7284.

12. F. Cazes, N. Moës, Comparison of a phase-field model and of a thick level set model for brittle and quasi-brittle fracture, Int. J. Numer. Meth. Engng. 103 (2015) 114-143.

13. D. Leguillon, Strength or toughness? A criterion for crack onset at a notch. Europ.J. Mech. A/Solids 21 (2002) 61-72.

14. Z. Yosibash, A. Bussiba, I. Gilad, Failure criteria for brittle elastic materials. Int. J. Fract. 125 (2004) 307-333.

15. L.M. Nguyen, D.Leguillon, O. Gillia, E. Riviere, Bond failure of a SiC/SiC brazed assembly, Mech. Mater.50 (2012) 1-8.

16. V. Mantič, Interface crack onset at a circular cylindrical inclusion under a remote transverse tension. Application of a coupled stress and energy criterion, Int. J. Solids Struct. 46 (2009) 287-1304

17. D. Leguillon, D. Quesada, C. Putot, E. Martin, Prediction of crack initiation at blunt notches and cavities - size effects, Eng. Fract. Mech. 74 (2007) 2420-2436.

18. E. Martin, D. Leguillon, N. Carrère, A twofold strength and toughness criterion for the onset of free-edge shear delamination in angle-ply laminates, Int. J. Solids Struct. 47 (2010) 12971305.

19. D. Leguillon, Determination of the length of a short crack at a v-notch from a full field measurement, Int. J. Solids Struct. 48(2011) 884-892.

20. P. Weissgraeber, D. Leguillon, W. Becker, A review of Finite Fracture Mechanics: Crack 
initiation at singular and non-singular stress-raisers. Archive Appl. Mech. 86 (2016) 375-401

21. B. Lawn, Fracture of Brittle Solids. Cambridge University Press, Cambridge, 1993.

22. V. Mantic, Prediction of initiation and growth of cracks in composites. Coupled stress and energy criterion of the finite fracture mechanics, Proceedings of the 16th European Conference on Composite Materials, June 2014.

23. L. Vincent, P. Soille, Watersheds in digital spaces: An efficient algorithm based on immersion simulations, IEEE PAMI, 13 (1991) 583-598.

24. B.T.M. Roerdink, A. Meijster, The Watershed Transform: Definitions, Algorithms and Parallelization Strategies, Fundamenta Informaticae 41 (2001) 187-228

25. J. Li, F. Song, C. Jiang, A non-local approach to crack process modeling in ceramic materials subjected to thermal shock, Eng. Fract. Mech. 133 (2015) 85-98.

26. P. Bouchard, F. Bay, Y. Chastel, Numerical modelling of crack propagation: automatic remeshing and comparison of different criteria, Comput. Methods Appl. Mech. Engng., 192 (2003) 3887-3908.

27. N. Moës, J. Dolbow, T. Belytschko. A finite element method for crack growth without remeshing, Int. J. Numer. Methods Engng 46 (1999) 131-150.

28. J. Li, X.B. Zhang, A criterion study for non-singular stress concentrations in brittle or quasibrittle materials, Engng Fract. Mech. 74, (2006) 505-523.

29. D. Leguillon, Z. Yosibash, Crack onset at a V-notch. Influence of the notch tip radius, International Journal of Fracture. 2003; 122: 1-21.

30. Tran V X, Leguillon D, Krishnan A, Xu L R, Interface crack initiation at V-notches along adhesive bonding in weakly bonded polymers subjected to mixed-mode loading, Int. J. Fract. 176 (2012) 65-79.

31. G. Sih, Handbook of stress-intensity factors, Lehigh University, 1973.

32. P. Judt, A. Ricoeur, Crack growth simulation of multiple cracks systems applying remote contour interaction integrals, Theor. Appl. Fract. Mech.,75 (2015) 78-88.

33. L. Ma, A. Korsunsky, On the use of vector J-integral in crack growth criteria for brittle solids, Int. J. Fract. 133 (2005) L39-L46.

34. A. Parvizi, K. Garrett, J. Bailey, Constrained cracking in glass fibre-reinforced epoxy crossply laminates. J. Mater. Sci. 13 (1978)195-201.

35. S.R. Kim, J. Nairn, Fracture mechanics analysis of coating/substrate systems, Part II: Experiments in bending, Engng. Fract. Mech. 65 (2000) 595-607 
Clean version

\title{
Finite element implementation of the coupled criterion for numerical simulations of crack initiation and propagation in brittle materials
}

\author{
Jia Li ${ }^{a^{*}}$, Dominique Leguillon ${ }^{\mathrm{b}}$
}

a LSPM, CNRS UPR 3407, Institut Galilée, Université Sorbonne Paris Cité, Université Paris 13, F93430 Villetaneuse, France

${ }^{\mathrm{b}}$ Institut Jean Le Rond d'Alembert, CNRS UMR 7190, Sorbonne Universités, UPMC Université Paris 06, F-75005 Paris, France.

*: Corresponding author

LSPM, CNRS UPR 3407

Institut Galilée, Université Paris 13

99 Avenue Jean-Baptiste Clément,

93430 Villetaneuse, France

Tel : $33149402889 \quad$ Fax : 33149403938

E-mail : jia.li@univ-paris13.fr

\begin{abstract}
The proposed numerical model for simulating two-dimensional cracking process in brittle materials is based on the coupled criterion (CC) which enters in the general framework of Finite Fracture Mechanics. It states that both the energy and stress conditions should be fulfilled to predict the crack creation in brittle solids. Special numerical approaches were developed in drawing correctly the potential crack paths and to minimize the potential energy with respect to all predicted crack paths. The originality of this work lies in the ability to describe both the initiation and the growth of one or more cracks. Moreover, statistical distributions of flaws can be accounted for. The efficiency and accuracy of the proposed approach were approved by means of several numerical examples for which the solutions are well-known or well-studied in the literature.
\end{abstract}

Keywords: Crack initiation, crack growth, multiple cracking, finite element analysis, brittle or quasi-brittle materials.

\section{Introduction}

Numerical prediction of fracture in solids is a classical challenge for mechanics and computational sciences. Developing efficient and accurate numerical models to simulate fracture behavior has been extensively studied in the last two decades. Even though numerous theoretical and numerical studies exist in the literature in predicting the crack growth, few numerical models are available to predict both the crack initiation and crack growth. 
Among these models, the most popular and efficient one nowadays is the cohesive zone model (CZM) [1-2]. A wide range of phenomena have been analyzed using this framework. Numerical schemes, like the finite element method, have become the mainstay for solution involving CZM. Crack propagation - crack nucleation can be modeled by the introduction of cohesive surfaces [3-4] allowing for shear as well as tensile debonding. One advantage of this type of formulation is that it no longer requires an initial crack, whose length and direction is difficult to prescribe, so that crack nucleation can easily be analyzed. Generally speaking, the cohesive elements can be inserted between all bulk elements such that the cohesive model can select automatically the crack paths. However, if the finite element mesh is refined and cohesive surfaces are continually added between all elements, convergence cannot always be expected. An additional result is logically the expensive computational costs. Practically, the cohesive elements are only inserted at predefined crack paths to avoid these numerical complications. These points have been well documented in the literature [5-6].

Apart from the CZM, the so-called phase-field model provides another possibility to predict both the crack initiation and crack growth. Francfort and Marigo [7] developed a so-called damage gradient model in which the damage zone converges to a crack as a prescribed length parameter tends to zero. Efficient algorithms were developed in order to solve complicated cracking problems [8-9]. Recently, phase-field models have found a widespread popularity for simulating brittle crack initiation and propagation in a smeared manner. However, concerns have been raised as well. It has been shown that the load-displacement curves can depend considerably on the internal length scale $l$ that defines the width of the distributed fracture zone and on the degradation function that has been introduced [10-11]. Once the parameters $G_{c}$, the critical energy release rate, and $l$ are specified according to the material under consideration and the mesh density is given, there is no room left to specify the critical stress [12].

In studying the crack onset from the root of a sharp V-notch, Leguillon [13] noticed that application of a single conventional fracture criterion, namely the Griffith energy criterion or the stress criterion, is not suitable and often leads to paradoxical predictions. It was concluded that when fracture occurs both criteria are fulfilled simultaneously, even if one often hides the other. Both are necessary conditions and together they form a sufficient one. Application of this coupled criterion (CC) to crack onset predictions at a V-notch root shows remarkably satisfactory agreement with respect to experimental results. Afterward, the $\mathrm{CC}$ gets increasing popularity in fracture prediction of brittle or quasi-brittle materials. A large number of fracture cases were studied by using this criterion [14-19 among others].

If the accuracy and efficiency of this criterion were approved by means of numerous real structure analyses, these studies were often performed case by case by using particular 
resolution techniques such as asymptotic analysis, local finite element modelling etc. Therefore, we believe that it is necessary and useful to implement this criterion into a numerical code such that more complicated fracture problems can be dealt with. This is the main motivation of the present work.

Herein, various numerical efforts were made for developing algorithms to predict automatically the location of crack nucleation and next their path. By means of these efforts, the $\mathrm{CC}$ was successfully implemented into a finite element code with which two-dimensional fractures in brittle materials can readily be simulated. Moreover, several classical fracture problems were studied in order to assess the efficiency of the proposed numerical model. The numerical results were compared with some well-known solutions in the literature to show that they are fully satisfactory.

\section{Description of the numerical fracture model}

\subsection{The coupled criterion}

The coupled criterion (CC) was widely presented in the literature (see the review paper by Weissgraeber et al. [20]). Herein we just give a brief outline. The CC is based on the Finite Fracture Mechanics (FFM) in which the Griffith criterion is written in incremental form:

$$
-\frac{\delta \Pi}{\delta S}=G^{i n c} \geq G_{c}
$$

where $\delta \Pi$ is the change in potential energy, $\delta S=t \delta l$ is the newly created crack surface (within the $2 \mathrm{D}$ framework $t$ is the thickness of the body under consideration and $\delta l$ the crack increment length), $G^{i n c}$ is the so-called incremental energy release rate and $G_{c}$ is its critical value representing the fracture toughness of the material. This criterion is not sufficient since it depends on the newly created surface $\delta S$ (up to now unknown). In order to remedy this shortcoming, the stress condition has to be added. For brittle materials, the maximal principal stress criterion is an appropriate candidate:

$$
\sigma_{1} \geq \sigma_{c}
$$

where $\sigma_{1}$ and $\sigma_{c}$ are respectively the maximal principal stress and the ultimate stress of the material. Within a plane elasticity framework, Leguillon [13] states that new crack is created if and only if both the stress and energy conditions are verified, i.e., the stress criterion is satisfied at all the points along the newly created crack path, i.e. over whole $\delta S$, and the Griffith criterion is satisfied with the corresponding crack path.

One of the main advantages of the $\mathrm{CC}$ is its ability to predict both the crack initiation and crack growth. In fact, through a matched asymptotic expansion analysis Leguillon [13] showed that the applied stress $\sigma_{a p p}^{*}$ at crack initiation can be written as 


$$
\sigma_{a p p}^{*}=E\left(\frac{G^{c}}{G_{0}}\right)^{1-\lambda}\left(\frac{\sigma^{c}}{\sigma_{0}}\right)^{2 \lambda-1}
$$

where $\lambda(<1)$ is the singularity exponent of the stress concentration (in case of a V-notch for instance), $\left(\sigma_{0}, G_{0}\right)$ are scaling coefficients. It is clear that this formulation coincides with the Griffith criterion in the presence of a crack $(\lambda=0.5)$ and with the strength criterion for a straight edge $(\lambda=1)$.

The CC is conceptually clear and physically convincing. Its application to different academic or engineering fracture problems has shown remarkable efficiency and accuracy. It is easy to apply when the fracture locations and the crack paths are previously known. However, these data are not always available for complex structures. Consequently, the first task in a numerical implementation of this criterion is to determine the locus of the crack creation. To this end, some simple but physically reasonable hypotheses have been adopted. In the following, we present the numerical details in the implementation of this criterion into a FE code. The three-node linear triangle elements are used in the simulations. Several reasons prompt us to use such a simple element. First, the adopted procedure of representing crack growth by element elimination requires very strong mesh refinements, thus it is convenient to use the simplest finite-element diminishing in this way high computational costs. Second, the usage of constant-stress finite-element allows a simple implementation of the procedure for crack path prediction proposed in Section 2.4. Finally, an increase of the degree of interpolation is useless in the vicinity of an elastic singularity because of the lack of smoothness of the solution.

\subsection{General computational consideration}

In the proposed numerical model, the external loads are applied in an incremental manner. According to the $\mathrm{CC}$, one of the necessary conditions of fracture is that the stress criterion $\sigma_{1} / \sigma_{c} \geq 1$ must be fulfilled all along the supposed crack paths. Consequently, we should firstly find all the zones in which $\sigma_{1} / \sigma_{c} \geq 1$, each containing one and only one local maximum of $\sigma_{1}$. We further assume that only one crack is created in each of such zones. If no such a zone is detected, the applied loads are incrementally increased.

The cracks may potentially initiate from the point where the first principal stresses $\sigma_{1}$ is locally maximum and propagates along the direction perpendicular to the first principal direction. Among all these potential cracks, the real cracks will be the combination making the incremental energy release rate maximum. This hypothesis is a classical one in fracture mechanics [21] even though the stress criterion is not considered. Furthermore, in the framework of FFM, Mantic [22] formulated mathematically this hypothesis as a minimization problem. All these hypotheses allow building a robust computational algorithm for the assessment of both cracks initiation and further cracks growth. 


\subsection{Locations of the potential newly created cracks.}

According to the above-mentioned general consideration, the first task is to find the zones in which $\sigma_{1} / \sigma_{c} \geq 1$, each containing a local maximum of $\sigma_{1}$ under a given prescribed load.

To this end, we inverse the sign of $\sigma_{1}$, such that a local maximum value become a local minimum value. Then we use the so-called watershed flooding technique to find these zones. The basic idea consists in considering the stress distribution as a topographic surface and placing a water source in each local minimum of its relief. Next the entire relief is gradually flooded from the sources and dams are placed where the different water sources meet. Figure 1 shows the flooding process of a 1D surface according to which the algorithm was built [23].

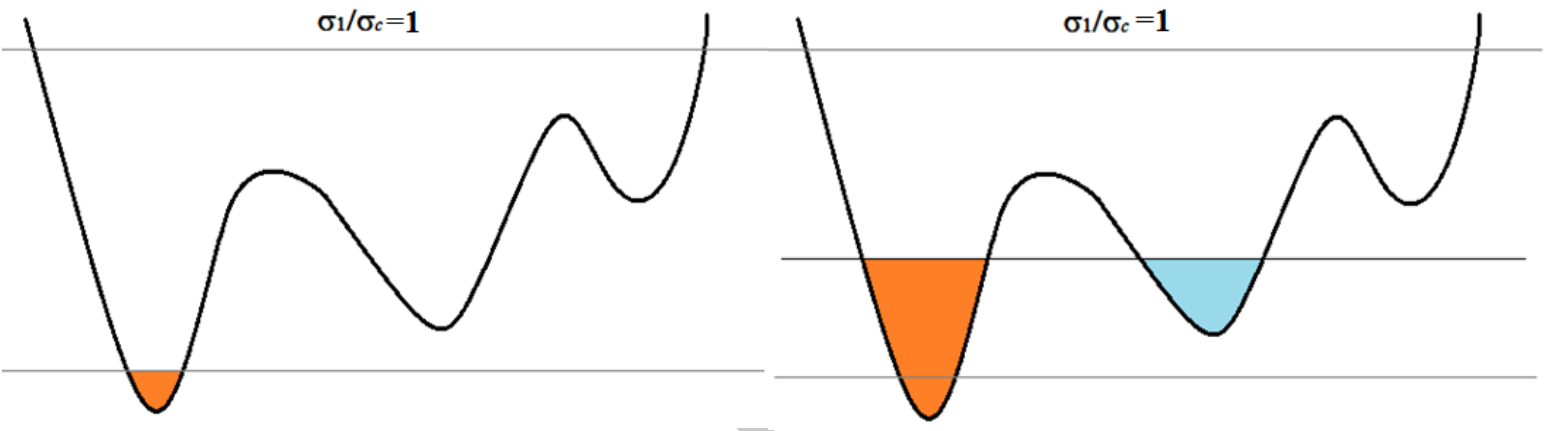

(b)

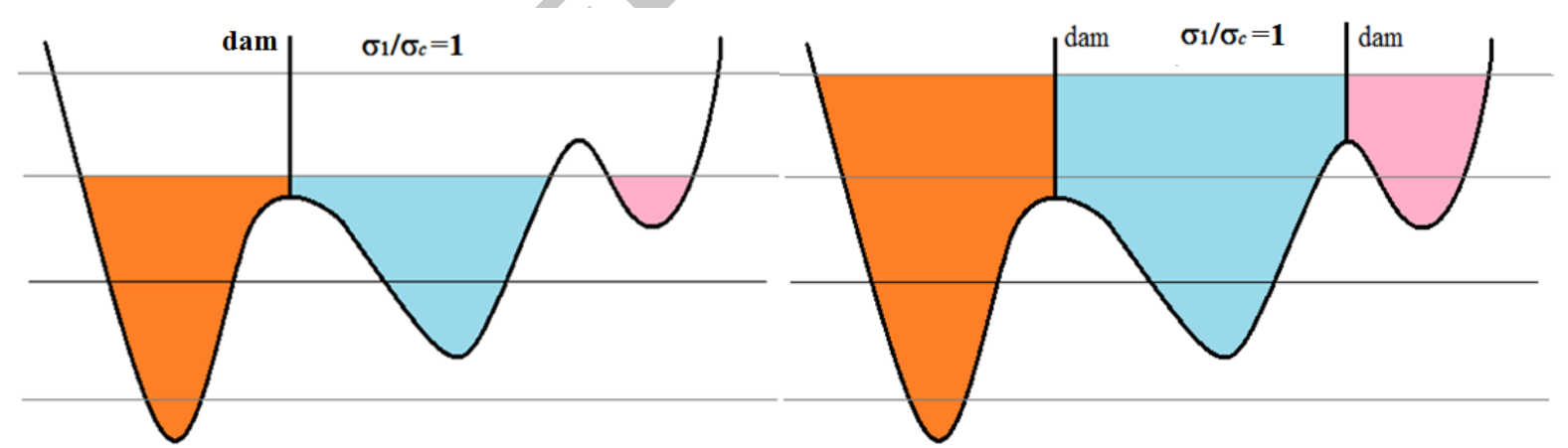

(c) (b)

(d)

Figure 1: Watershed flooding process (a): a water source is placed at the point where $-\sigma_{1} / \sigma_{c}$ is minimum, increasing the water level allows finding the neighboring elements and then creating the first catchment basin; (b) by increasing the water level, another distinguished local minimum is found from which the second catchment basin is created; (c) when the first and second catchment basins meet, a dam is placed; (d) continuing this process until $\sigma_{1} / \sigma_{c}=1$ allows finding distinct catchment basins containing each a local minimum of $-\sigma_{1} / \sigma_{c}$.

All elements in the zone around a given minimum constitute the catchment basin associated with that minimum. The watersheds are the zones dividing adjacent catchment basins. The 
first points that are reached by water are the elements at the highest stress value, i.e., points where $-\sigma_{1} / \sigma_{c}$ are minimal, and then all elements are progressively reached up to the stress level $\sigma_{1} / \sigma_{c}=1$.

The catchment basins are labeled with integer values. Each catchment basin contains a local maximum $\sigma_{1}$ from which a potential crack can be created.

Normally, the classical watershed technique often lead to over-segmentation phenomenon, which consists in finding out too many catchment basins due to noisy numerical results. There exist numerous denoising techniques in the literature to reduce this over-segmentation [24]. In this work, we just pre-process the topographic surface by using the median filtering technique for its simplicity. The main idea of the median filter is to run through the stress distribution map element by element, replacing the $\sigma_{1}$ value of the element with the median value of neighboring elements. In the case when the stress evaluation is sufficiently accurate, this simple technique is sufficient to reduce efficiently the over-segmentation phenomenon.

This algorithm allows extracting $N$ zones $\mathbf{S}_{n}$, each of them containing a local maximum of the first principal stress like a "hill peak".

\subsection{Drawing the potential crack paths}

In each zone $\mathbf{S}_{n}$ a potential crack can be created. According to the maximal principal stress criterion, the direction of the newly created crack is perpendicular to the first principal stress. This criterion permits to draw a principal stress trajectory from each "hill peak". The following algorithm is used to determine the crack paths:

6. From the point $\left(x_{0}, y_{0}\right)$ corresponding to the "hill peak" in $\mathbf{S}_{n}$, we draw first the right branch of the predicted crack path. To this end, we calculate the coordinates $x_{1}=x_{0}+\Delta l \cos \alpha_{0}, y_{1}=y_{0}+\Delta l \sin \alpha_{0}$, where $\alpha_{0}$ is the angle of the first principal stress trajectory at the point $\left(x_{0}, y_{0}\right)$. Varying $\Delta l$ if necessary such that the point $\left(x_{1}, y_{1}\right)$ is located in an adjacent element;

7. If the point $\left(x_{1}, y_{1}\right)$ is not located in $\mathbf{S}_{n}$, go to step 5. Else from the point $\left(x_{1}, y_{1}\right)$ and with the angle of the first principal stress trajectory $\alpha_{1}$ at this point, calculate

$x_{2}^{\text {right }}=x_{1}+\Delta l \cos \alpha_{1}, y_{2}^{\text {right }}=y_{1}+\Delta l \sin \alpha_{1}$ and

$x_{2}^{\text {left }}=x_{1}-\Delta l \cos \alpha_{1}, y_{2}^{\text {left }}=y_{1}-\Delta l \sin \alpha_{1}$

Varying $\pm \Delta l$ if necessary such that the points $\left(x_{2}^{\text {right }}, y_{2}^{\text {right }}\right)$ and $\left(x_{2}^{\text {left }}, y_{2}^{\text {left }}\right)$ are located in the adjacent elements;

8. Let $x_{2}=x_{2}^{\text {right }}, y_{2}=y_{2}^{\text {right }}$ if $\sigma_{1}\left(x_{2}^{\text {right }}, y_{2}^{\text {right }}\right)>\sigma_{1}\left(x_{2}^{\text {left }}, y_{2}^{\text {left }}\right)$, else let $x_{2}=x_{2}^{\text {left }}, y_{2}=y_{2}^{\text {left }}$;

9. Let $x_{1}=x_{2}, y_{1}=y_{2}$ and go to step 2 until $\left(x_{2}, y_{2}\right)$ is no longer located in $\mathbf{S}_{n}$.

10. From the point $\left(x_{0}, y_{0}\right)$, draw the left branch of the predicted crack path by following the same algorithm as above-described. 
For each zone $\mathbf{S}_{n}$, a potential crack path is drawn. Simultaneously, the length of the crack path can be obtained by cumulating $\Delta l$.

\subsection{Creation of cracks by using the maximum energy release rate criterion}

It is clear that even all the predicted crack paths can be drawn by using the above-described algorithm, they will probably not all be activated to become real cracks. Which ones of them become real cracks depend on the fulfillment of the energy criterion $-\delta \Pi / \delta S=G^{i n c} \geq G_{c}$.

All the combinations of the possible crack extensions should be considered in order to find the maximal energy release rate. If we have predicted $N$ potential crack paths and each path may become real crack or not, $2^{N}$ combinations are possible to become real cracks. Consequently, $2^{N}$ linear elastic finite element resolutions have to be performed: one for the structure before crack extension, and $2^{N}-1$ for the structure with all the possible crack extension combinations. The combination which makes $G^{i n c}$ maximum and verifies the Griffith criterion $G^{i n c} \geq G_{c}$ will become real cracks.

For the creation of the new cracks, different approaches exist in the framework of the finite element method. We believe that the algorithm proposed above allows using the following techniques:

4. Remove the elements along the predicted crack paths [25];

5. Remesh the elements near the predicted crack path by creating a new crack [26];

6. Introduce discontinuities along the predicted crack paths [27].

It is clear that amongst all these methods the first one is the simplest. For this reason it was used in the present work. In practice, we find all elements in interception with the predicted crack paths, and then remove them from the finite element model. In return, this obviously requires a sufficiently fine mesh to be as close as possible to the concept of crack.

\subsection{Overall algorithm}

In order to avoid complicated snap-back analyses and to guarantee the existence of the solutions, the external loads are applied incrementally by imposing displacements on the boundaries of the considered structure until a prefixed level. By using the techniques described above, we can construct an overall algorithm as follows:

8. At each loading step, a first finite element calculation is carried out to determine the stress distribution and the total potential energy $\Pi_{0}$ in the structure;

9. With the obtained stress distribution and by using the watershed flooding process, we first find $N$ zones $\mathbf{S}_{n}$ in which $\sigma_{1} \geq \sigma_{c}$ and containing each a local maximum of $\sigma_{1}$;

10. If $N=0$, increase the external loads and go to step 1 .

11. Else, in each such zone $\mathbf{S}_{n}$, a potential crack path is drawn by following a principal stress trajectory; 
12. By activating or not each potential crack path, perform $2^{N}-1$ finite element calculations for all possible combinations and find the corresponding total potential energies $\Pi_{i}$ and the crack length increment $\Delta l_{i}$. For each combination, we calculate the incremental energy release rate $G_{i}^{i n c}=-\frac{\Pi_{i}-\Pi_{0}}{\Delta l_{i}} ;$

13. Consider the crack path having the maximal incremental energy release rate $G_{i}^{\text {inc }}$. If the energy criterion $\max \left(G_{i}^{i n c}\right) \geq G_{c}$ is fulfilled, create the corresponding new cracks by eliminating all elements along the predicted crack paths. Go to step 1;

14. Go to step 1 without increasing the external loads.

\section{Validation and numerical examples}

In order to validate the proposed numerical fracture model, we carry out numerical simulations on some special structures for which the fracture modes are well-known or wellstudied in the literature. Through these examples, the efficiency and the accuracy of the model, especially the mesh independency and the capacity in modelling the multiple cracking processes, are also discussed.

\section{(1): Crack initiation from a hole in a plate}

An earlier experimental work [28] showed that the strength of a drilled plate depends closely on the hole size. It was shown that this size effect can be captured by using the coupled criterion. In this work, we will evaluate the remote loads at fracture of the specimens by using the present numerical tool and compare them with the theoretical predictions, obtained by creating an ideal crack from the hole boundary and calculating its exact stress intensity factor according to the CC. Since the material used in the experiments, the polymethyl metacrylate (PMMA), is a very brittle material, the crack initiation leads to immediate structure failure of the specimens. Therefore, the remote load at the crack onset is considered as the strength of the drilled plate.

The mechanical characteristics of the material are as follows: Young's modulus $E=3000$ $\mathrm{MPa}$, Poisson's ratio $v=0.36$; the ultimate tensile stress $\sigma_{c}=72 \mathrm{MPa}$; the critical energy release rate $G_{c}=290 \mathrm{~J} / \mathrm{m}^{2}$. The section of the specimens is of $10 \times 30 \mathrm{~mm}^{2}$. The strengths of the plate with holes of different sizes are evaluated by using the present numerical method. In order to evaluate the influence of the mesh size on the crack onset prediction, three different element sizes near the hole are used in the simulations, namely $d=0.005,0.01$ and $0.02 \mathrm{~mm}$, where $d$ is the average edge length of the triangular elements. It is useful to note that the characteristic length of the material is given by $\left(K_{c} / \sigma_{c}\right)^{2} / 2 \pi=0.026 \mathrm{~mm}$.

The numerical predictions of the strength for different hole sizes are plotted in Figure 2. The ordinate axis represents $\sigma_{\infty} / \sigma_{c}$, the ratio of the remote tensile stress at fracture over the ultimate tensile stress. For comparison, the theoretical predictions obtained by using the $\mathrm{CC}$ $[13,28]$ are also plotted. From this figure, we can remark that the numerical strength 
predictions agree very well with the theoretical ones for fine meshes, i.e., for $d=0.005$ and $0.01 \mathrm{~mm}$. For the coarser mesh $d=0.02 \mathrm{~mm}$, the agreement is still good when the hole diameter is larger than $1 \mathrm{~mm}$. The numerical predictions become slightly more conservative than the theoretical ones when the hole diameter is smaller than $1 \mathrm{~mm}$. As expected, $\sigma_{\infty}$ tends to $\sigma_{c}$ as the hole size tends to zero, it tends to $\sigma_{c} / 3$ as the hole is large.

The main difference between the theoretical and numerical approaches remains in the fact that the creation of ideal cracks in the theoretical approach is replaced by the removed elements in the numerical one. The decrease of the total potential energy is more important in the case when deleting elements, which leads to an over-estimation of the incremental energy release rate. Consequently, the prediction of the crack onset from a non-cracked structure is more conservative by using the element elimination technique. This error can be reduced by using refined mesh. From Figure 2, we can remark that the mesh sizes $d=0.005$ and $0.01 \mathrm{~mm}$ give very satisfactory crack onset predictions comparing to the theoretical predictions. We can also observe that when the mesh is sufficiently refined, the crack onset prediction is very weakly mesh-dependent. It is also worthy to note that the element elimination technique is much simpler to implement in a numerical code than the creation of real cracks, especially for multi-cracking problems.

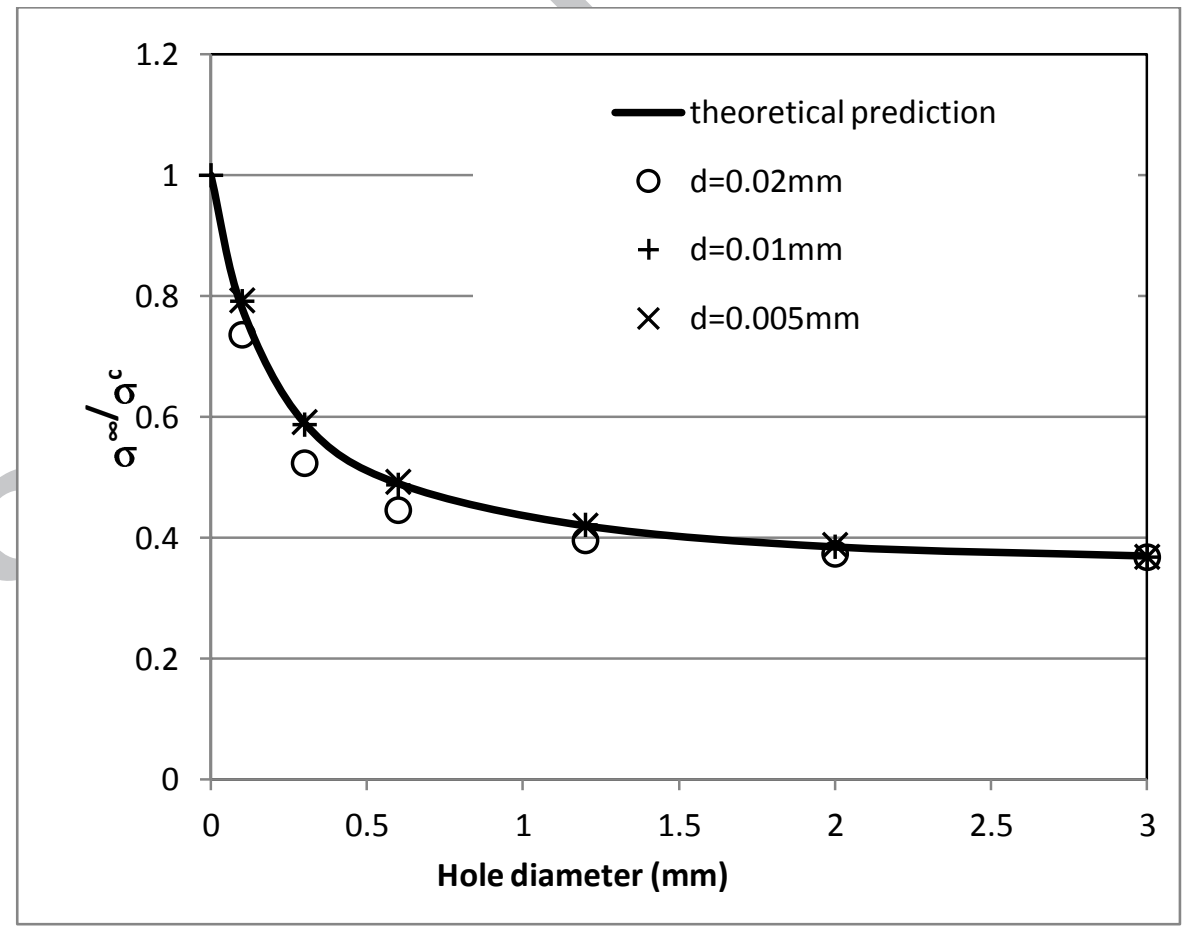

Figure 2: Critical remote stress at fracture of the drilled specimens as function of the hole size. Comparison between the theoretical and numerical predictions

Figure 3 shows the first steps of the crack initiation and growth from the hole boundary. The elements in which the stress condition $\sigma_{1} \geq \sigma_{c}$ is fulfilled are drawn in green. The predicted 
crack path is plotted in black line. The elements drawn in red are at the intersection with the predicted crack path and have to be deleted. The black band represents the already removed elements. The crack onset is predicted according to the CC, i.e., both the stress and energy criteria are fulfilled, that explains the quite lengthy crack initiation (about $0.07 \mathrm{~mm}$, Fig. 3a). After the initiation, the crack grows rapidly under fixed remote displacement loading and it follows unstable crack propagation (Fig. 3b-3c) as observed in the experiments [28].
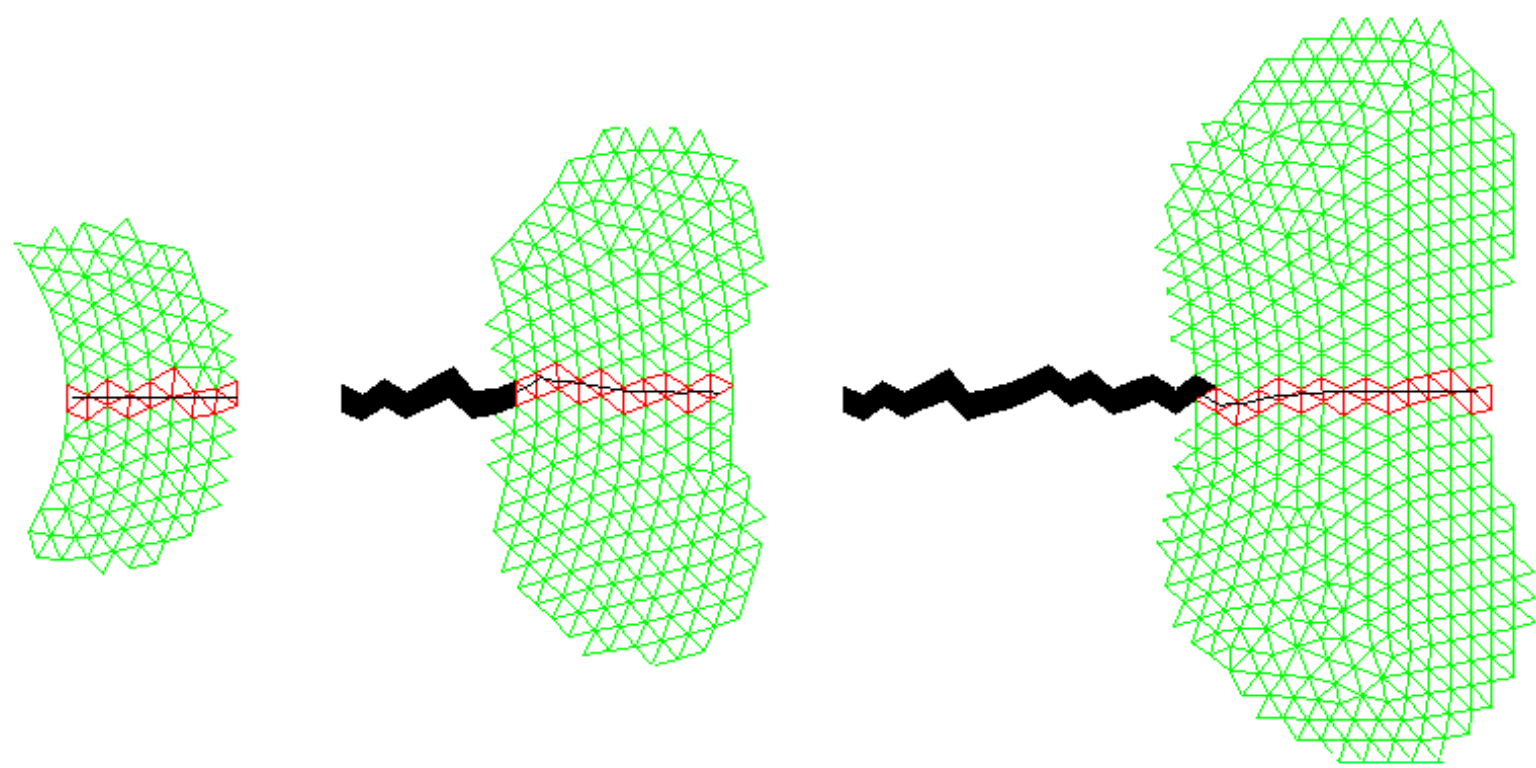

(b)

(b)

(c)

Figure 3: Numerical simulation of the crack initiation and growth (a): crack onset from the hole boundary (b)-(c): crack growth under unchanged remote displacement load

\section{(2): Crack initiation from the tip of a $V$-notch}

Leguillon [13] performed a detailed study on crack onset from the tip of a sharp V-notch, on the basis of which the $\mathrm{CC}$ was proposed. By using the $\mathrm{CC}$, the remote loads at fracture, the crack direction and the crack onset length were successfully predicted. In fact, the asymptotic stress along the crack path can be written as

$$
\sigma\left(r, \theta_{0}\right)=k r^{\lambda-1} s\left(\theta_{0}\right)
$$

where $\theta_{0}$ is the crack orientation with respect to the $\mathrm{V}$-notch bisector; $r$ is the distance between the $\mathrm{V}$-notch tip and a point at the crack path. The singularity exponent is such that $1 / 2 \leq \lambda \leq 1$ and $k$ is the generalized stress intensity factor. The potential energy change at a crack onset is written:

$$
-\delta \Pi=k^{2} K\left(\theta_{0}\right) l^{2 \lambda} t
$$


where $t$ is the thickness of the specimen; $l$ is the crack onset length and $K$ is a scaling term depending on the opening angle and the crack direction. The use of the CC (i.e. (4) for $0 \leq r \leq$ $l$ and (5)) leads to the crack onset criterion in terms of the generalized stress intensity factor:

$$
k \geq k_{c} \quad \text { with } \quad k_{c}=\left(\frac{G_{c}}{K\left(\theta_{0}\right)}\right)^{1-\lambda}\left(\frac{\sigma_{c}}{s\left(\theta_{0}\right)}\right)^{2 \lambda-1}
$$

In [13], the coefficients $k$ and $K$ were evaluated by means of the matched asymptotic procedure. The crack direction $\theta_{0}$ maximizes the denominator in the right hand side of (6).

Hereafter we evaluate the remote loads at crack onset of a V-notched specimen and compare the results with Leguillon's predictions. The geometry and the loading condition of the specimen are illustrated in Figure 4. The thickness of the specimen is $e=5 \mathrm{~mm}$. Displacements are prescribed at its top and bottom ends. The material parameters are as follows: Young's modulus $E=3000 \mathrm{MPa}$, Poisson's ratio $v=0.36$; the ultimate tensile stress $\sigma_{c}=72 \mathrm{MPa}$; the critical energy release rate $G_{c}=290 \mathrm{~J} / \mathrm{m}^{2}$. The $\mathrm{V}$-notch opening angle is $2 \beta$ $=90^{\circ}$. Fine mesh is used around the V-notch tips with the element size $d=0.005 \mathrm{~mm}$. Three loading cases, the pure tension, the pure shear and a mixed mode, are studied (Table 1). Eqn. (6) can be used in the two first cases, considering either the first (symmetric) or second (antisymmetric) mode of the Williams expansion associated with two different exponents $\lambda$ (Table 1). In the more entangled mixed mode, an extension of (6) must be used, taking into account together the two above mentioned modes. See [29,30] for the generalization of (6) to complex loadings.

The remote prescribed displacements $u_{1}$ or $u_{2}$ are incrementally increased until the CC is fulfilled. The critical remote resulting forces $F_{1}$ for shear and $F_{2}$ for tension obtained in this way are compared with the results using Leguillon's method, as shown in the following table:

Table 1: Critical remote loads at crack initiation of V-notched specimens

\begin{tabular}{|l|l|l|l|}
\hline $\begin{array}{l}\text { Prescribed } \\
\text { displacements }\end{array}$ & $\begin{array}{l}u_{1}=0 \text { and } u_{2} \neq 0 \\
\text { pure tension }\end{array}$ & $\begin{array}{l}u_{1} \neq 0 \text { and } u_{2}=0 \\
\text { pure shear }\end{array}$ & $\begin{array}{l}u_{1} / u_{2}=10 \\
\text { mixed mode }\end{array}$ \\
\hline Leguillon's method & $\lambda=0.545$ & $\lambda=0.906$ & $\lambda_{1}=0.545, \lambda_{2}=0.906$ \\
& $\theta_{0}=0 \mathrm{deg}$. & $\theta_{0}=57 \mathrm{deg}$. & $\theta_{0}=35 \mathrm{deg}$. \\
& $F_{1}=0$ & $F_{1}=10819 \mathrm{~N}$ & $F_{1}=4731 \mathrm{~N}$ \\
& $F_{2}=2048 \mathrm{~N}$ & $F_{2}=0$ & $F_{2}=1752 \mathrm{~N}$ \\
\hline $\begin{array}{l}\text { Present numerical } \\
\text { method }\end{array}$ & $\theta_{0}=0 \mathrm{deg}$. & $\theta_{0}=54 \mathrm{deg}$. & $\theta_{0}=31 \mathrm{deg}$. \\
& $F_{1}=0$ & $F_{1}=10931 \mathrm{~N}$ & $F_{1}=4611 \mathrm{~N}$ \\
& $F_{2}=2082 \mathrm{~N}$ & $F_{2}=0$ & $F_{2}=1746 \mathrm{~N}$ \\
\hline
\end{tabular}

This comparison shows that the numerical predictions agree very well with the matched asymptotic procedure proposed in [13]. The differences on the critical remote loads are limited within $2 \%$. The agreement between the crack directions may seem less accurate but it 
must be pointed out that it is difficult to measure in the numerical simulation, where the crack has a somewhat saw-tooth appearance caused by the removed elements.

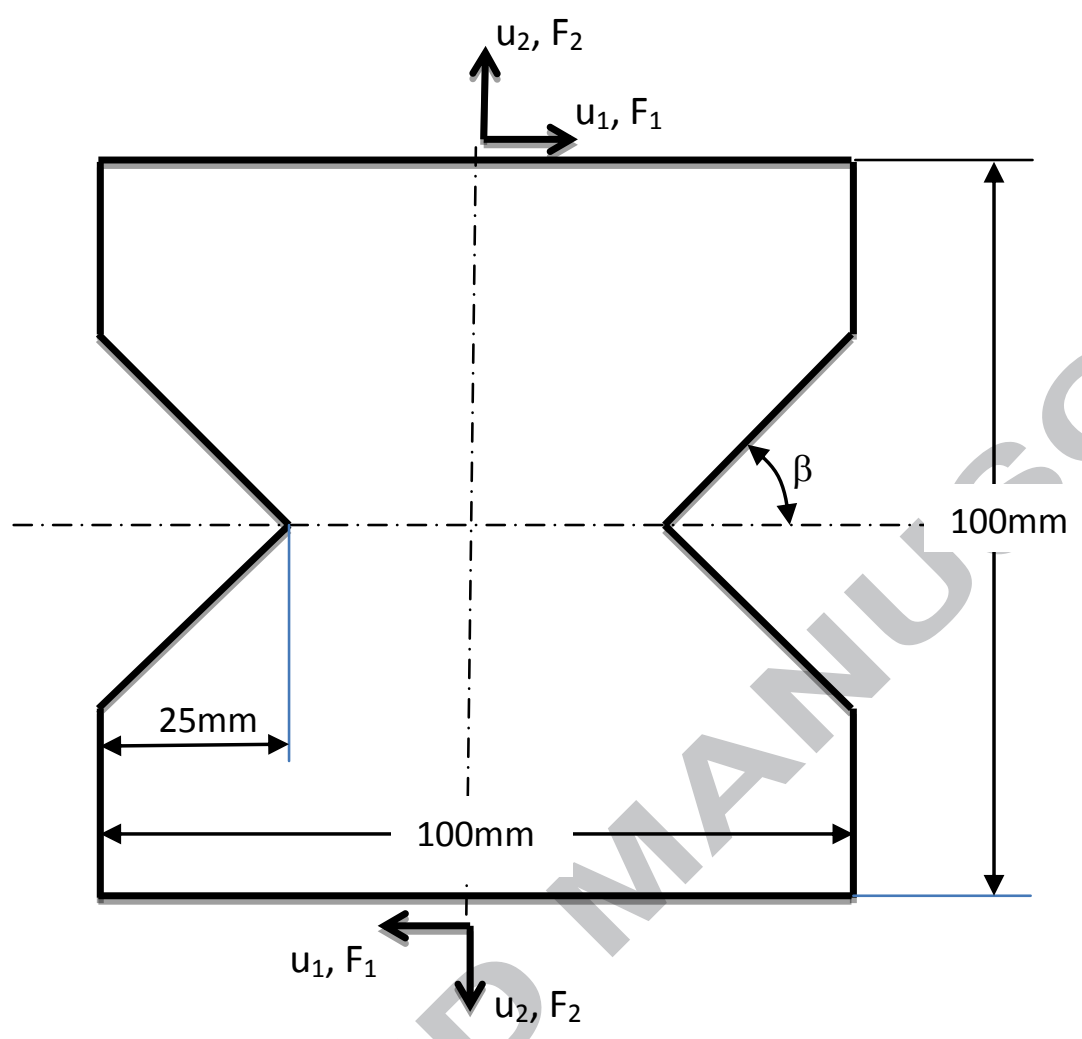

Figure 4: Geometry and load conditions of the V-notched specimen

\section{(3): Crack initiation and growth}

The third example in the validation of the present model is a plate including a central crack subjected to a pure mode-I remote loading. We will show that in this case, the CC is equivalent to the Griffith criterion if the pre-existing crack is long enough.

Consider a 2D square plate with a central crack of length $2 a$. The dimension of the specimen is $2 W \times 2 W=10 \times 10 \mathrm{~mm}^{2}$. We evaluate the critical remote loads for crack growth with a central crack of different sizes, namely $a / W=0,0.02,0.04,0.06,0.08,0.1,0.2,0.3,0.4$ and 0.5 , here $a=0$ means that there is no crack in the plate. The mechanical properties of the material are: Young's modulus $E=3000 \mathrm{MPa}$, Poisson's ratio $v=0.3$, ultimate stress $\sigma_{c}=$ $150 \mathrm{MPa}$, critical stress intensity factor $K_{c}=67 \mathrm{MPa} \sqrt{\mathrm{mm}}$. By considering the symmetry, only one half of the plate is modeled. In order to investigate the influence of the mesh size, different element sizes near the crack tip are used in the simulations, namely $d=0.003,0.006$ and $0.01 \mathrm{~mm}$, where $d$ is the average edge length of the triangular elements. A uniaxial tension along the direction normal to the crack was applied at the ends of the plate by imposing incrementally increasing displacements. The remote load is applied gradually until the CC is verified. Figure 5 shows the fracture details near the crack tip. 


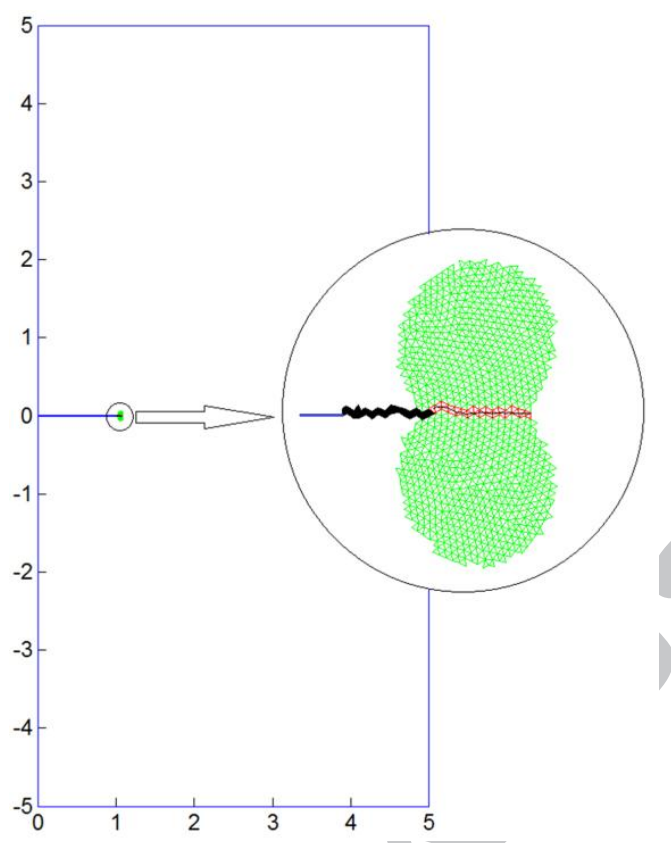

Figure 5: Growth simulation of a central crack. The black band represents the removed elements after the first step of the crack growth.

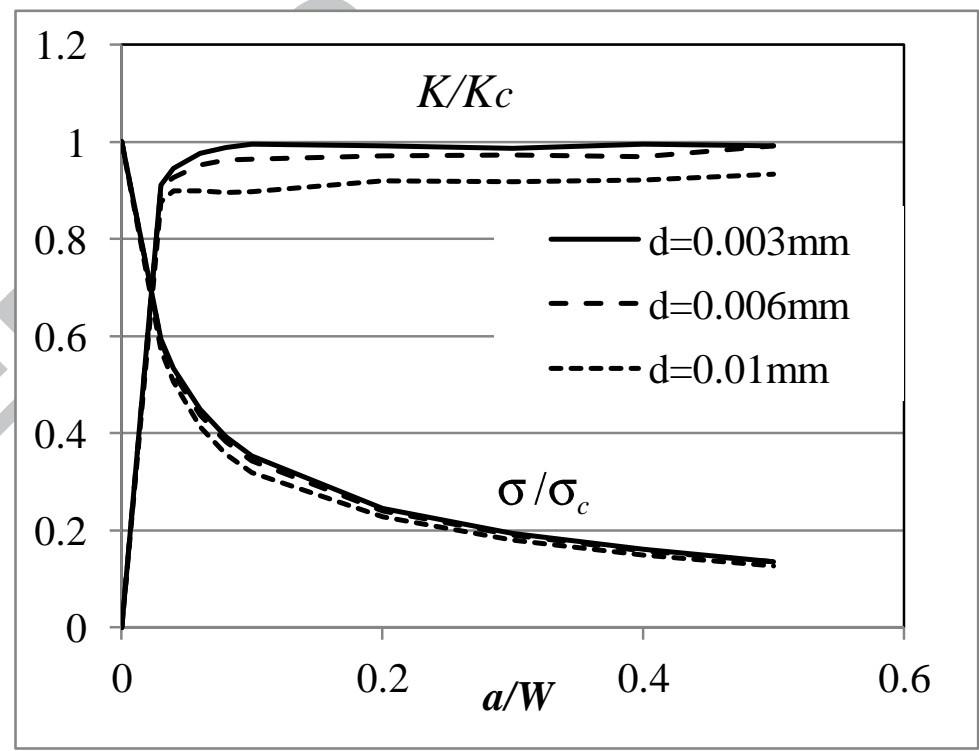

Figure 6: Normalized stress intensity factor $K_{\mathrm{I}} / K_{\mathrm{Ic}}$ and normalized remote stresses $\sigma^{\infty} / \sigma_{c}$ as a function of the normalized semi-crack length $a / W$ for mode-I loaded cracks at fracture.

The critical remote load at crack propagation thus obtained was used to calculate the stress intensity factor by using a formula found from a handbook of stress intensity factors [31]. By comparing the obtained stress intensity factors to the critical value $K_{c}$, we can evaluate the accuracy of the method for crack growth prediction. The results of these calculations are 
shown in Figure 6. From this figure, we can observe that for sufficiently long cracks $(a / W>$ $0.05)$, the normalized stress intensity factor $K / K_{c} \approx 1$. Therefore, the stress intensity factors at fracture evaluated by using the $\mathrm{CC}$ equal correctly the material fracture toughness. For very short cracks, the Griffith fracture criterion is no longer sufficient to predict the crack growth. The critical stress intensity factor decreases as the crack length decreases. Moreover, the remote stress at fracture tends to the material tensile strength as the crack length tends to zero. In this case, the $\mathrm{CC}$ degenerates to the maximum principal stress criterion for nonsingular stresses.

We can observe that the crack growth prediction is very weakly mesh-sensitive when sufficiently refined meshes are used. Apart from the results calculated with $d=0.01 \mathrm{~mm}$ that represents quite a coarse mesh, the calculations with finer meshes, e.g. $d=0.003 \mathrm{~mm}$ and $0.006 \mathrm{~mm}$, provide us with very close values for remote loads at fracture. In fact, the predicted crack path should contain sufficient number of elements in order to guarantee a good accuracy on the stress and energy calculations. For crack growth, the length of predicted crack path can be calculated by $l=\left(K_{c} / \sigma_{c}\right)^{2} / 2 \pi \approx 0.032 \mathrm{~mm}$ for the used material with the parameters above-mentioned. From Figure 6, we remark that the element size $d=l / 5$ offers already sufficient accuracy for the crack growth prediction. The complete mesh-independency can be expected by using very refined meshes, which will increase drastically the computational costs.

\section{(4): Crack path tracking: interaction of two cracks}

The present method enables efficient crack path tracking when the stress field is accurately evaluated. We illustrate this feature by the following example. Consider a rectangular plate with two non-symmetric cracks at its side edges subjected to tensile loading (Figure 7). We calculate the critical remote load at fracture and the crack paths by using the proposed method and then compare them with the results found in the literature. Recently, Judt and Ricoeur [32] studied this problem by using a variant of the $J$-integral technique [33]. The geometry of the specimen is shown in Figure 7. The material parameters are chosen as follows: Young's modulus $E=72000 \mathrm{MPa}$, Poisson's ratio $v=0.33$, ultimate stress $\sigma_{c}=300 \mathrm{MPa}$ and material fracture toughness $K_{c}=23.9 \mathrm{MPa} \sqrt{\mathrm{m}}$.

It is clear that the initial geometrical symmetry of the crack positions is unstable. From experimental or numerical point of view, a small perturbation can break this symmetry such that one of the cracks can first grow while the other remains arrested. In order to keep approximately the geometrical symmetry during the crack propagation, we enforce the simultaneous growth of the two cracks, even though the minimum potential principle may be slightly violated.

In order to assess the mesh-sensitivity of the method, we performed simulations with 3 element sizes, namely $d=0.075 \mathrm{~mm}, 0.1 \mathrm{~mm}$ and $0.15 \mathrm{~mm}$. In Figure 7 , we show the crack paths obtained with the different meshes. For comparison, the crack paths obtained in [32] are 
also plotted in this figure. We can see that the three meshes provide almost the same crack paths which agree very well with the result presented in [32]. Here again, the mesh-sensitivity is shown very weak with the adopted meshes and the accuracy of the proposed model is satisfactory in predicting the crack paths.

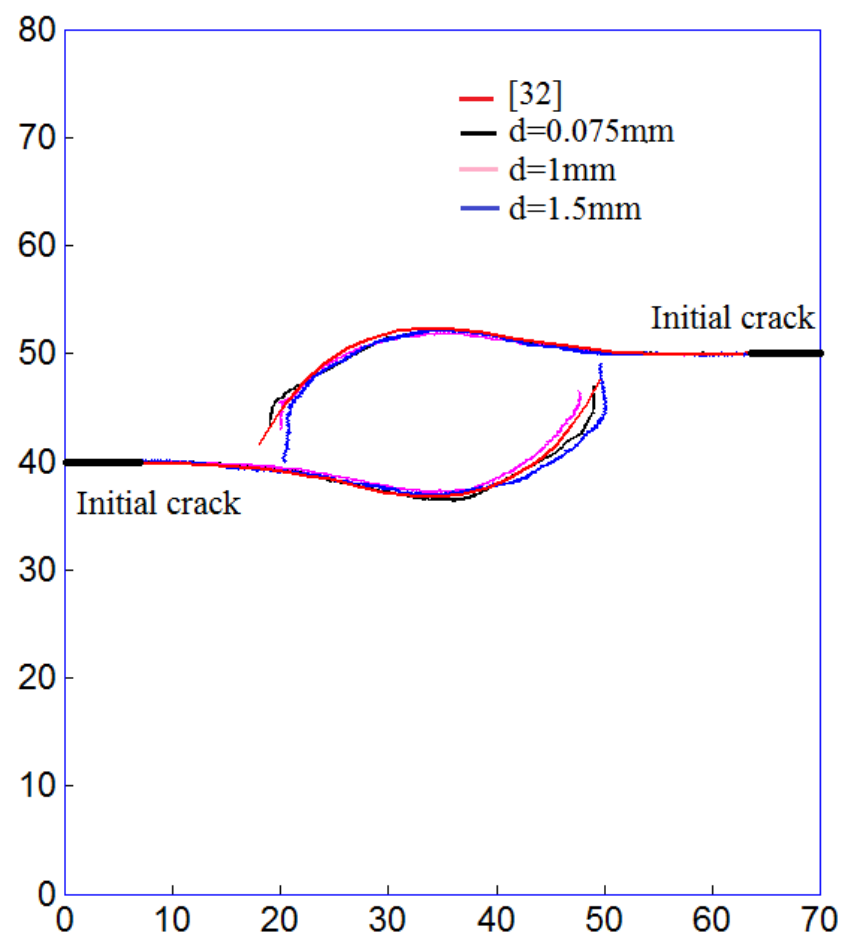

Figure 7: Crack paths predicted by using different meshes in a plate with double cracks

\section{(5): Multi-cracking: Cracking in fiber-reinforced epoxy cross-ply laminates}

Multiple crack initiations and growths can occur simultaneously. Simulation of multiple cracking processes is a big challenge for computational mechanics. The proposed numerical model is able to deal with this problem in a rigorous manner. In fact, all the possible new crack loci are examined by applying the $\mathrm{CC}$ and the best combination of them that makes the total potential energy minimal is selected as the next cracks extensions.

In the following, we consider the experimental works performed by Parvizi et al. [34] on the transverse fracture of glass fiber-reinforced cross-ply laminates. The numerical simulations of their experiments can be used to validate Leguillon's criterion and also, to show the ability of the proposed method in modelling the multi-cracking process.

Parvizi et al. [34] carried out tensile tests on a cross-ply glass fiber-reinforced composite made of two outer layers oriented at $0 \circ$ (fibers are parallel to the tension direction) and an inner one oriented at $90^{\circ}$, as shown in Figure 8 . The thickness of the inner ply was varied and the applied strain at the first occurrence of a transverse crack was recorded. As the applied load increases, multiple cracks were observed in the inner ply. 


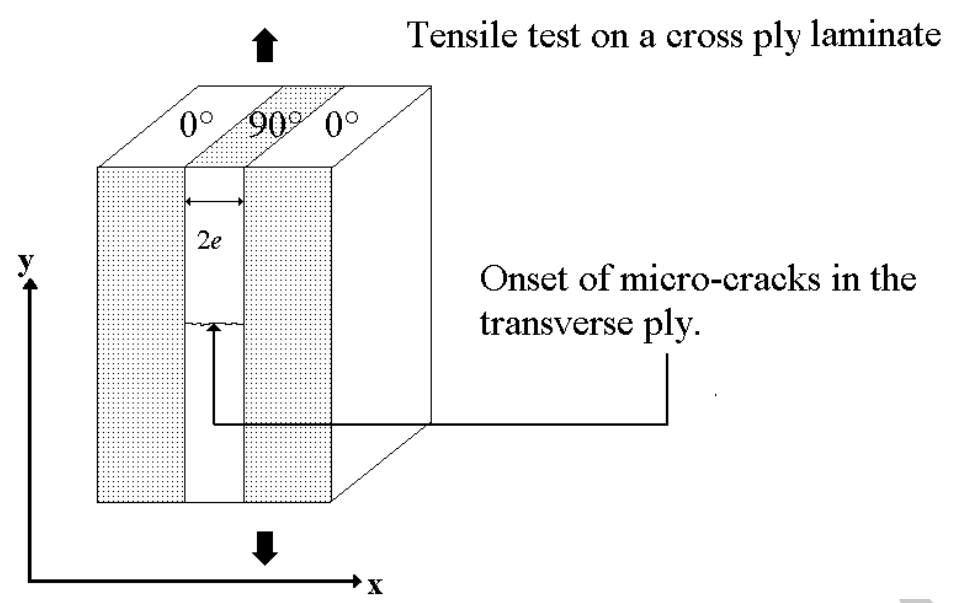

Figure 8: Scheme of the tensile tests on a cross-ply glass fiber-reinforced composite Include the

The samples were modeled by using bi-dimensional plane-strain triangle elements. Only one half of the sample was meshed by symmetry. The plies were regarded as orthotropic materials with the following Hooke's law:

$$
\left\{\begin{array}{l}
\sigma_{x x} \\
\sigma_{y y} \\
\sigma_{x y}
\end{array}\right\}=\left[\begin{array}{ccc}
\frac{E_{x}}{1-v_{x}^{2}} & \frac{v_{x} E_{x}}{1-v_{x}^{2}} & 0 \\
\frac{v_{x} E_{x}}{1-v_{x}^{2}} & \frac{E_{y}}{1-v_{y}^{2}} & 0 \\
0 & 0 & \mu_{x y}
\end{array}\right]\left\{\begin{array}{c}
\varepsilon_{x x} \\
\varepsilon_{y y} \\
\gamma_{x y}
\end{array}\right\}
$$

According to the data given in [32], the mechanical properties of the outer ply are: $E_{x}=14000$ $\mathrm{MPa} ; E_{y}=42000 \mathrm{MPa} ; v_{x}=0.13 ; v_{y}=0.4 ; \mu_{x y}=10000 \mathrm{MPa}$, and for the inner ply : $E_{x}=E_{y}=$ $14000 \mathrm{MPa} ; v_{x}=v_{y}=0.13 ; \mu_{x y}=6194 \mathrm{MPa}$. The outer ply was considered as unbreakable. For the inner ply, according to [34], we took $\sigma_{c}=84 \mathrm{MPa}$ and $G_{c}=0.12 \mathrm{MPa} \mathrm{mm}$. The length of the samples is taken to be sufficiently long $(L=10 \mathrm{~mm})$ such that the appearance of the first crack is not influenced by the length of the samples. The thickness of the outer ply is $0.5 \mathrm{~mm}$. Several thicknesses of the inner ply were considered in the simulation, namely $2 e=0.05,0.1$, $0.2,0.3,0.4,0.5,1,4 \mathrm{~mm}$ respectively. The specimens were subjected to uniaxial tension in the vertical direction by imposing incrementally increasing displacements on the outer layers of the composite.

We first illustrate how the proposed method initiates the first crack in the inner ply. Figure 9-a shows the distribution of the first principal stresses in the inner ply just before the onset of the first crack. In Figure 9-b, we plot the first principal stresses of the inner ply along the interface. From these two figures, we can find that the stress concentrations are of a very high level at the two ends of the specimen near the interface. At the center of the specimen, it appears a very large zone in which the first principal stress exceeds the material strength with a nearly uniform distribution. The proposed algorithm should choose the location of the first 
crack initiation according to the CC. In fact, three zones including a local stress maximum were first found by applying the watershed line method, as shown in Figure 9-c. Then the potential crack paths were created from the local stress maxima by following the direction normal to the first principal stress. As shown in Figure 9-d, although the stress concentration is much higher at extremities than in the central part of the specimen, the potential crack paths are much shorter. Consequently, these potential cracks have small impact on the total potential energy so that the energy criterion is not fulfilled. On the contrary, the potential crack at the central part of the specimen is much longer. Its activation provokes a much larger variation of the total potential energy. Consequently, both the stress and energy criteria are fulfilled to create first a new crack at the central part of the specimen. This is what was observed in the experiments [34].

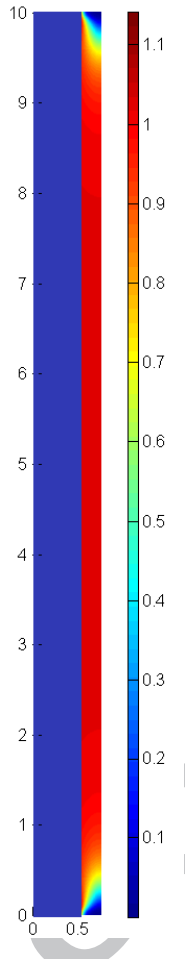

(b)

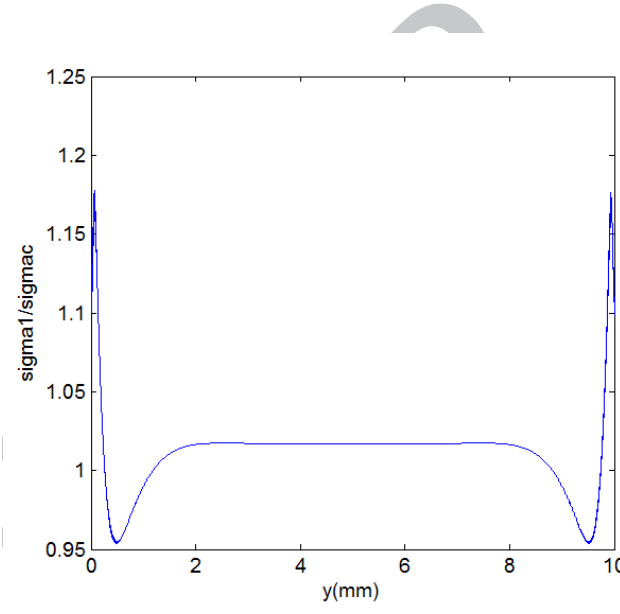

(b)

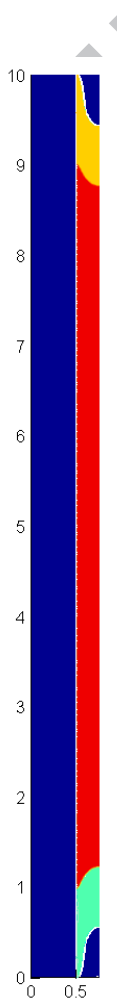

(c)

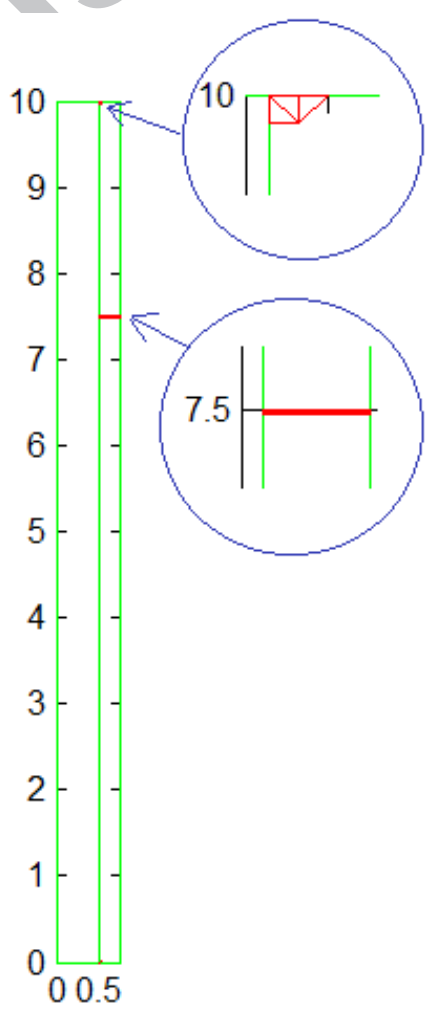

(d)

Figure 9: Stress distribution and the potential crack paths. (a) Distribution of $\sigma_{1} / \sigma_{c}$; (b) Variation of $\sigma_{1} / \sigma_{c}$ along the interface; (c) Three zones found in which there is a local maximum of $\sigma_{1} / \sigma_{c} \geq 1$; (d) Potential crack paths

By following this method, we simulated the experiments for specimens of different thicknesses of the inner layer. The imposed longitudinal strains at first crack initiation were obtained, then compared with the experimental data of Parvizi et al., as shown in Figure 10. We can remark a very satisfactory agreement between the numerical and experimental results. 
In fact, for specimens with a thin inner ply, the energy criterion governs the failure while the stress criterion is trivially fulfilled. The contrary occurs for specimens with a thick inner ply.

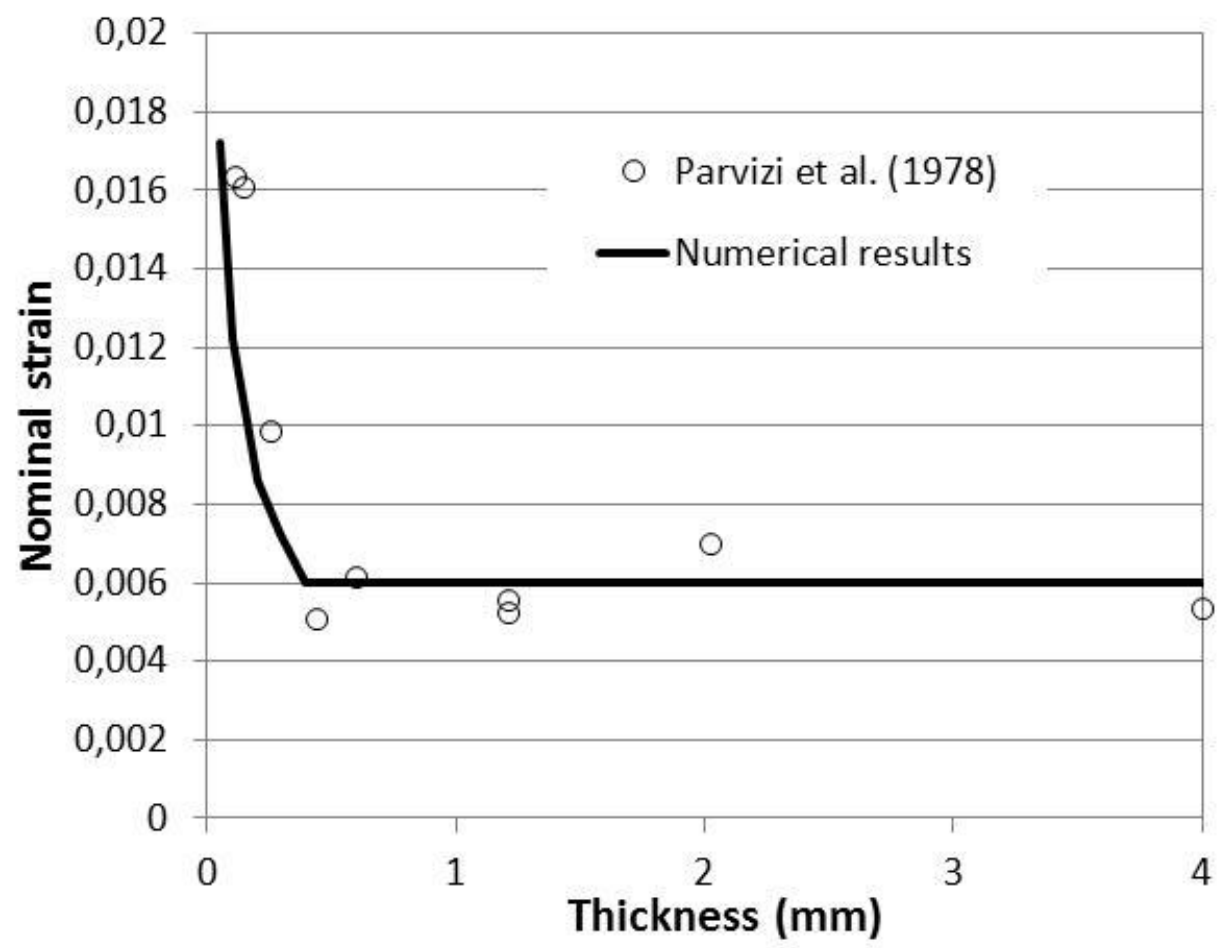

Figure 10: Nominal strain at the occurrence of the first transverse crack versus the thickness of the inner ply

It is clear that one of the interesting features of the proposed method is its ability to simulate the multi-cracking process in composite materials for instance. The further crack creation process in the laminate was simulated for different thicknesses of the inner ply. In Figure 11, we plot the density of the transverse cracks in the inner ply as a function of the prescribed strain. As expected, specimens with a thin inner ply sustain higher loads for the first crack formation. However, the crack density increases rapidly as the load increases. For specimens with a thick inner ply, the first crack formation requires smaller remote loads. As the stress relaxation is larger when creating a longer crack, the further crack formation requires higher remote loads. This trend was also observed in some experimental works [35] on thin coatings. 


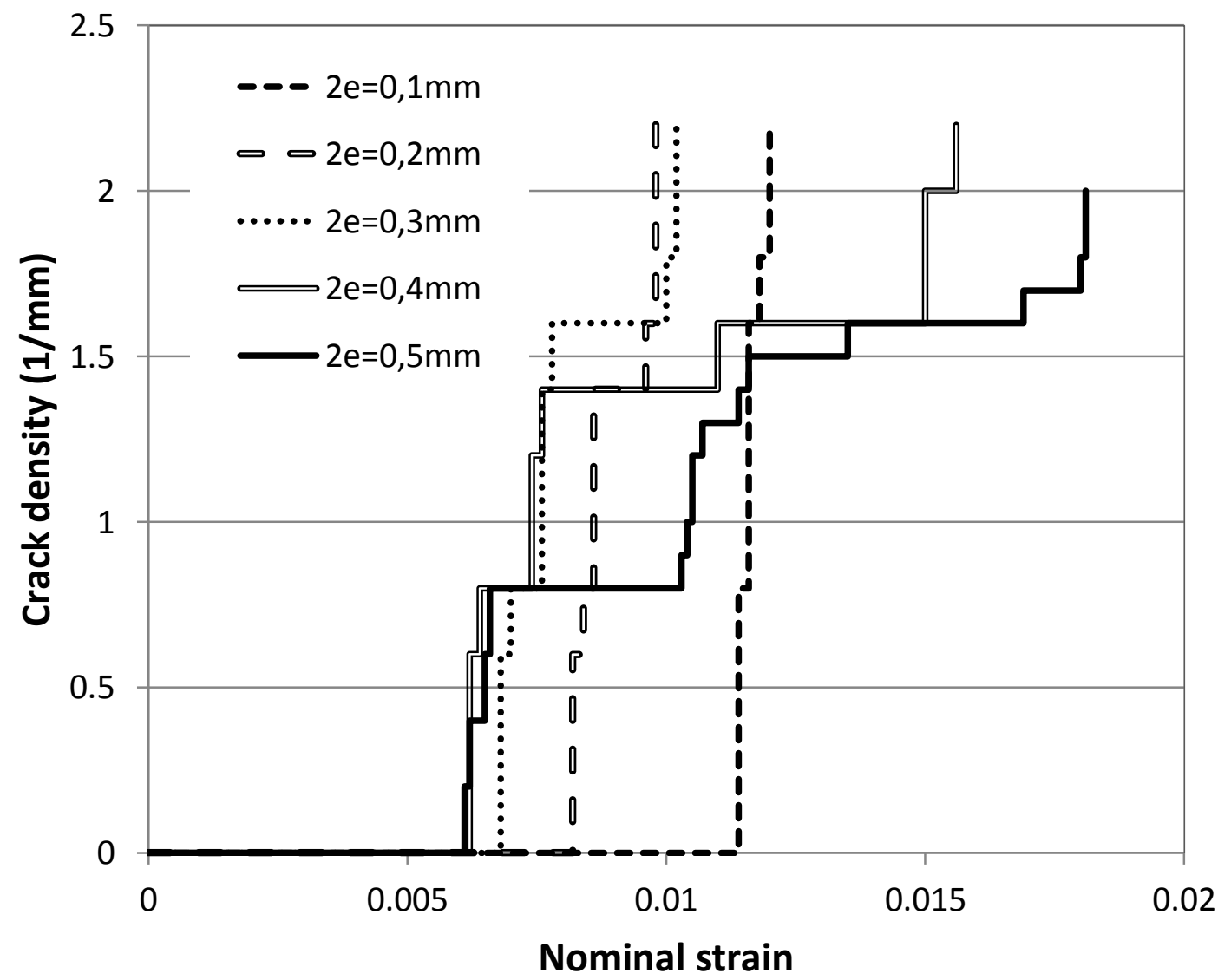

Figure 11: Crack density as function of Nominal strain for different thickness of the inner ply

\section{(6): Cracking in composite materials}

In this section, we attempt to assess the multi-cracking process in composite materials. In this case, the strengths and the fracture toughness are function of the spatial coordinates. In this case, the watershed technique permits to create automatically distinct potential cracks in adjacent materials of the composite. According to the CC, we have to ensure that $-\delta \Pi / \delta S$ is maximal for the crack combination. However, a global energy criterion $-\delta \Pi / \delta S \geq G_{c}$ does not exist for creating several cracks simultaneously as the values of $G_{c}$ may be different for each crack. In order to overcome this difficulty, we assume furthermore that the energy criterion $-\delta \Pi^{(i)} / \delta S^{(i)} \geq G_{c}^{(i)}$ is fulfilled for all the individual cracks of the combination, where $\delta \Pi^{(i)}, \delta S^{(i)}, G_{c}^{(i)}$ are respectively potential energy change, the newly created crack surface and the critical energy release rate when only the crack $(i)$ is activated in a single material component of the composite.

To illustrate this feature, we consider again the fiber-reinforced laminate formerly treated in example (5) by taking some scattering into account. In the following simulations, we assume that the strength and the fracture toughness are randomly distributed in the inner ply of the 
composite following a Weibull distribution. The two parameters Weibull's law used in this work writes:

$$
f\left(\sigma_{c}\right)=\frac{m}{\sigma}\left(\frac{\sigma_{c}}{\sigma}\right)^{m-1} e^{-\left(\frac{\sigma_{c}}{\sigma}\right)^{m}}
$$

where $f\left(\sigma_{c}\right)$ stands for the failure probability at $\sigma_{1}=\sigma_{c}, m$ and $\sigma$ are the Weibull parameters, with $m=9$ and $\sigma=89 \mathrm{MPa}$. Following this probability function, the material strength is randomly distributed in the inner ply by spots of $0.5 \mathrm{~mm} \times e$. The fracture toughness of each spot is related to the strength by $G_{c}=0.132\left(\sigma_{c} / \sigma\right)^{2} \mathrm{MPa}$.mm. These strength and fracture toughness distributions will generate approximately a mean strength of $84 \mathrm{MPa}$ and a mean fracture toughness of $0.12 \mathrm{MPa} . \mathrm{mm}$ as those in example (5). One of the strength distributions and the frequency histogram are shown in Figure 12.

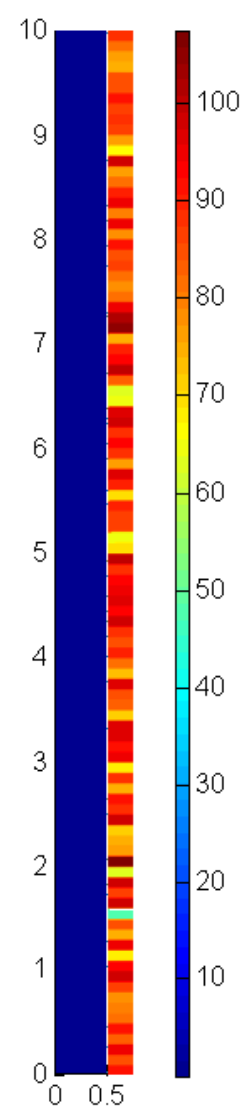

(a)

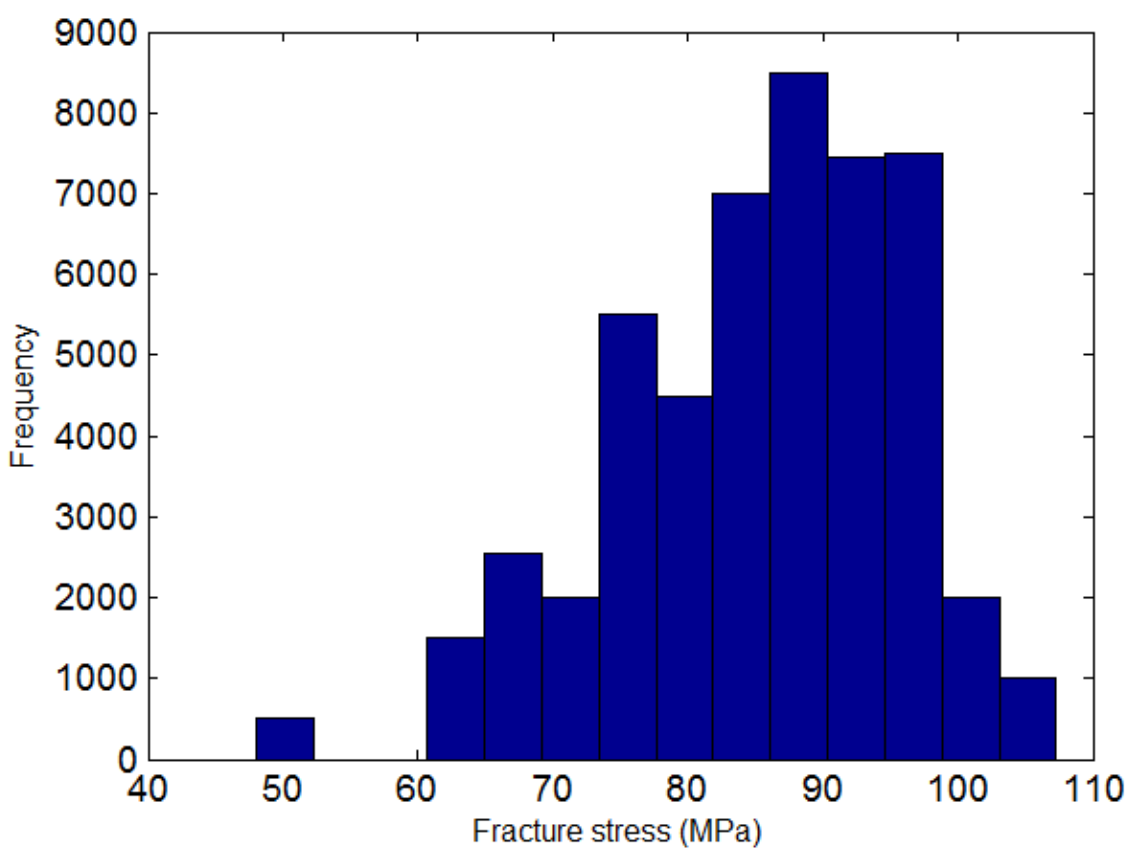

(b)

Figure 12: (a) Strength distribution in the inner ply according to the Weibull model (b)

Histogram of strength

By means of this probabilistic approach, the previous numerical simulations were revisited. The numerical results are plotted in Figure 13. Comparing to Figure 11, the curves of crackdensity vs. nominal strain show more graduate variation with a gentle slope. These results exhibit a better similarity to those obtained in experiments [35]. 
Figure 14 shows the crack patterns in the inner ply obtained by using the deterministic and probabilistic approaches respectively. Noting that the crack pattern of the deterministic model is very regular; the cracks appear by following nearly a dichotomy rule, while the probabilistic model provides a more irregular crack pattern. We can also note that small cracks emerge when the crack density reaches a critical level for both the deterministic and probabilistic models. In real composite materials, the interface debonding is often observed instead of the small transverse cracks. As the interface debonding is not taken into account in the present work, the small transverse cracks appear in the numerical simulations.

This example shows numerous capacities of the proposed method in simulating multicracking process in heterogeneous materials. As our attention is focused on the methodology description, detailed comparison with experimental works has not been realized.

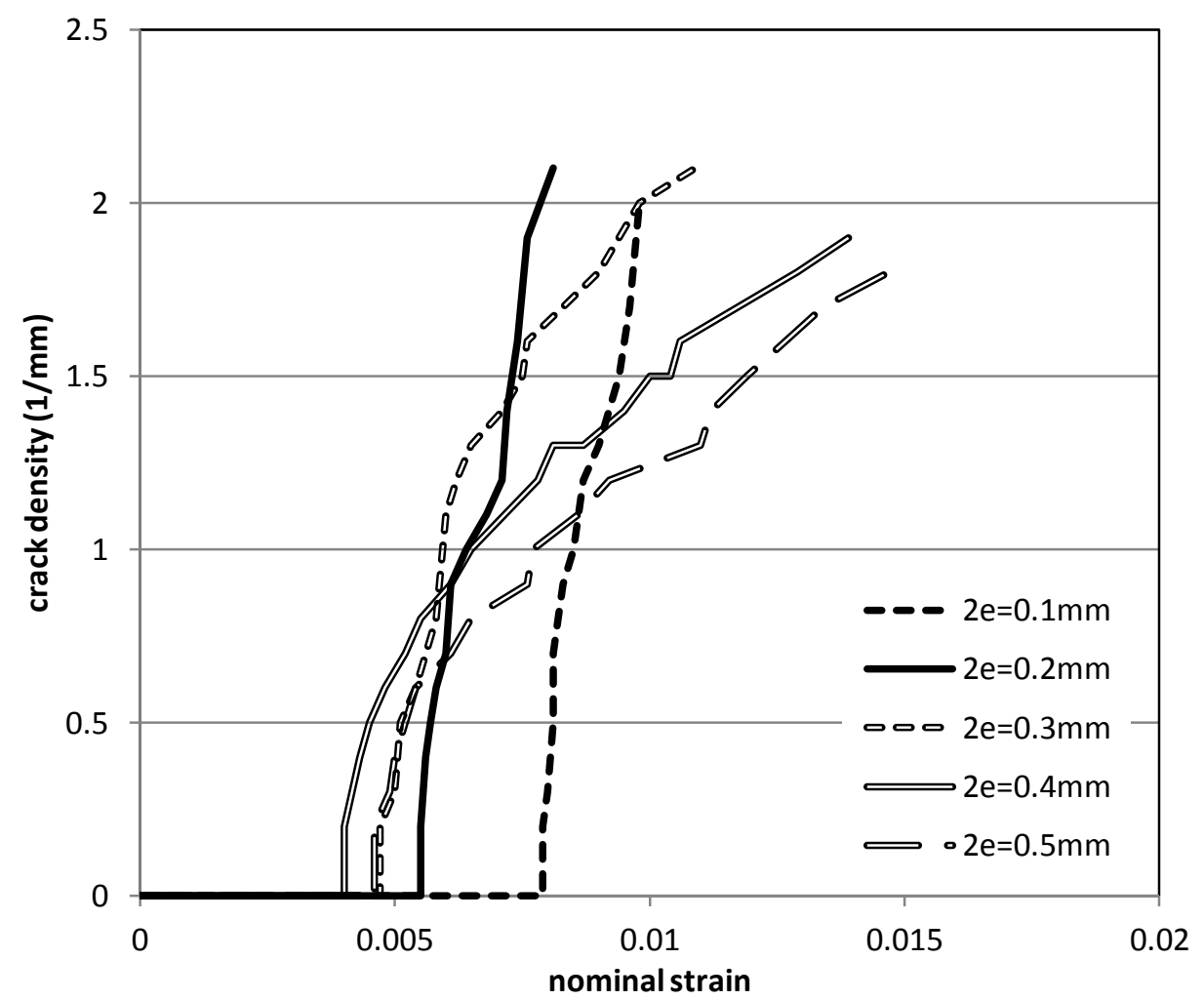

Figure 13 Crack density as function of Nominal strain for different thickness of the inner ply according to the Weibull model 

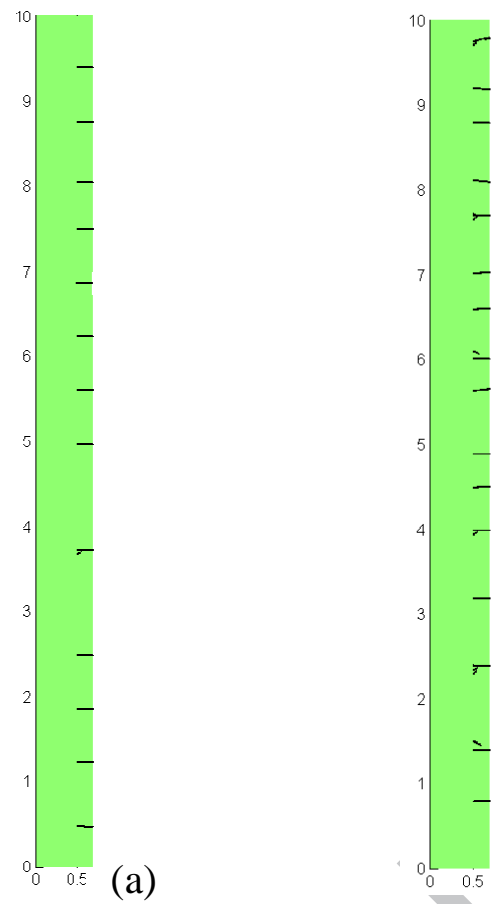

(b)

Figure 14: Crack patterns in the inner ply obtained by using the deterministic (a) and probabilistic (b) approaches respectively at nominal strain $\varepsilon=0.0115$

\section{Conclusions}

In this work, we have implemented the fracture criterion proposed by Leguillon [13] into a finite element code for simulating two-dimensional cracking process in brittle materials. By means of a large number of examples for which the solutions are well-known or well-studied in the literature, the validity of the proposed numerical model was confirmed. It was shown that this numerical model presents several advantages, for example:

- Both crack initiation and crack growth can be predicted;

- Multi-cracking problems can easily be considered;

- Cracking process in heterogeneous materials can be simulated;

- Once the finite element model provides sufficient accuracy on stress evaluation, the fracture prediction is nearly mesh-insensitive.

- Convergence can always be guaranteed if displacement boundary conditions are prescribed.

- Only linear elastic calculations are required. This allows building a robust and efficient computational algorithm.

It is clear that further researches are necessary to improve the numerical model. One of the tasks is to build smooth stress fields in order to avoid the over-segmentation problem that leads to too many potential cracks. In this topic, the denoising techniques developed in image processing science [24] should be more thoroughly investigated. Another drawback of the model may be its huge computational costs required for a large number of cracks. Even though parallel computing techniques can largely increase the calculation speed, 
supplementary hypotheses and more efficient algorithms are expected to reduce the computational tasks.

The couple criterion for fracture prediction is conceptually simple and physically reasonable. Its numerical implementation opens a new way to describe the cracking process in solids. We believe that many other complex problems in fracture evaluations can be treated by developing more operational numerical tools.

\section{References}

1. G.I. Barenblatt, The mathematical theory of equilibrium cracks in brittle fracture, Adv. Appl. Mech. 7 (1962) 55-129.

2. D. Dugdale, Yielding of steel sheets containing slits, J. Mech.Phys.Solids, 8(2) (1960) 100104.

3. X.P. Xu, A. Needleman, Numerical Simulation of Fast Crack Growth in Brittle Solids. J. Mech. Phys. Solids 42 (1994) 1397-1434.

4. G.T. Camacho, M. Ortiz, Computational modelling of impact damage in brittles materials, Int. J. Solids Struct. 33 (1996) 2899-2938.

5. A. Needleman, V. Tvergaard, Finite element analysis of localization in plasticity. In: J.T. Oden and G.F. Carey (Eds.), Finite elements, Special problems in solid mechanics, PrenticeHall, New Jersey, 1984 pp. 94-157.

6. A. Needleman, Some issues in cohesive surface modeling, 23rd International Congress of Theoretical and Applied Mechanics, Procedia IUTAM 10 ( 2014 ) 221-246.

7. G. Francfort, J.J. Marigo, Revisiting brittle fracture as an energy minimization problem. J. Mech. Phys.Solids 46 (1998) 1319-1342.

8. B. Bourdin, G. Francfort, J.J Marigo, Numerical experiments in revisited brittle fracture. J. Mech. Phys. Solids 48 (2000) 797-826.

9. H. Amor, J.J. Marigo, C. Maurini , Regularized formulation of the variational brittle fracture with unilateral contact: numerical experiments. J. Mech. Phys.Solids 57 (2009)1209-1229.

10. J. Vignollet, S, May, R. de Borst, C.V. Verhoosel, Phase-field models for brittle and cohesive fracture. Meccanica 49 (2014) 2587-2601.

11. S. May, J. Vignollet, R. de Borst, A numerical assessment of phase-field models for brittle and cohesive fracture: G-Convergence and stress oscillations, Europ. J. Mech. A/Solids 52 (2015) 7284.

12. F. Cazes, N. Moës, Comparison of a phase-field model and of a thick level set model for brittle and quasi-brittle fracture, Int. J. Numer. Meth. Engng. 103 (2015) 114-143.

13. D. Leguillon, Strength or toughness? A criterion for crack onset at a notch. Europ.J. Mech. A/Solids 21 (2002) 61-72.

14. Z. Yosibash, A. Bussiba, I. Gilad, Failure criteria for brittle elastic materials. Int. J. Fract. 125 (2004) 307-333.

15. L.M. Nguyen, D.Leguillon, O. Gillia, E. Riviere, Bond failure of a SiC/SiC brazed assembly, Mech. Mater.50 (2012) 1-8.

16. V. Mantič, Interface crack onset at a circular cylindrical inclusion under a remote transverse tension. Application of a coupled stress and energy criterion, Int. J. Solids Struct. 46 (2009) 287-1304

17. D. Leguillon, D. Quesada, C. Putot, E. Martin, Prediction of crack initiation at blunt notches and cavities - size effects, Eng. Fract. Mech. 74 (2007) 2420-2436.

18. E. Martin, D. Leguillon, N. Carrère, A twofold strength and toughness criterion for the onset of free-edge shear delamination in angle-ply laminates, Int. J. Solids Struct. 47 (2010) 12971305.

19. D. Leguillon, Determination of the length of a short crack at a v-notch from a full field measurement, Int. J. Solids Struct. 48(2011) 884-892.

20. P. Weissgraeber, D. Leguillon, W. Becker, A review of Finite Fracture Mechanics: Crack 
initiation at singular and non-singular stress-raisers. Archive Appl. Mech. 86 (2016) 375-401

21. B. Lawn, Fracture of Brittle Solids. Cambridge University Press, Cambridge, 1993.

22. V. Mantic, Prediction of initiation and growth of cracks in composites. Coupled stress and energy criterion of the finite fracture mechanics, Proceedings of the 16th European Conference on Composite Materials, June 2014.

23. L. Vincent, P. Soille, Watersheds in digital spaces: An efficient algorithm based on immersion simulations, IEEE PAMI, 13 (1991) 583-598.

24. B.T.M. Roerdink, A. Meijster, The Watershed Transform: Definitions, Algorithms and Parallelization Strategies, Fundamenta Informaticae 41 (2001) 187-228

25. J. Li, F. Song, C. Jiang, A non-local approach to crack process modeling in ceramic materials subjected to thermal shock, Eng. Fract. Mech. 133 (2015) 85-98.

26. P. Bouchard, F. Bay, Y. Chastel, Numerical modelling of crack propagation: automatic remeshing and comparison of different criteria, Comput. Methods Appl. Mech. Engng., 192 (2003) 3887-3908.

27. N. Moës, J. Dolbow, T. Belytschko. A finite element method for crack growth without remeshing, Int. J. Numer. Methods Engng 46 (1999) 131-150.

28. J. Li, X.B. Zhang, A criterion study for non-singular stress concentrations in brittle or quasibrittle materials, Engng Fract. Mech. 74, (2006) 505-523.

29. D. Leguillon, Z. Yosibash, Crack onset at a V-notch. Influence of the notch tip radius, International Journal of Fracture. 2003; 122: 1-21.

30. Tran V X, Leguillon D, Krishnan A, Xu L R, Interface crack initiation at V-notches along adhesive bonding in weakly bonded polymers subjected to mixed-mode loading, Int. J. Fract. 176 (2012) 65-79.

31. G. Sih, Handbook of stress-intensity factors, Lehigh University, 1973.

32. P. Judt, A. Ricoeur, Crack growth simulation of multiple cracks systems applying remote contour interaction integrals, Theor. Appl. Fract. Mech.,75 (2015) 78-88.

33. L. Ma, A. Korsunsky, On the use of vector J-integral in crack growth criteria for brittle solids, Int. J. Fract. 133 (2005) L39-L46.

34. A. Parvizi, K. Garrett, J. Bailey, Constrained cracking in glass fibre-reinforced epoxy crossply laminates. J. Mater. Sci. 13 (1978)195-201.

35. S.R. Kim, J. Nairn, Fracture mechanics analysis of coating/substrate systems, Part II: Experiments in bending, Engng. Fract. Mech. 65 (2000) 595-607

Highlights

- A new numerical fracture model for brittle materials was established on the basis of the coupled criterion;

- Both crack initiation and crack growth in complex 2D structures can accurately be predicted;

- Multiple cracking processes can be simulated.

- The fracture predictions are weakly mesh-sensitive. 